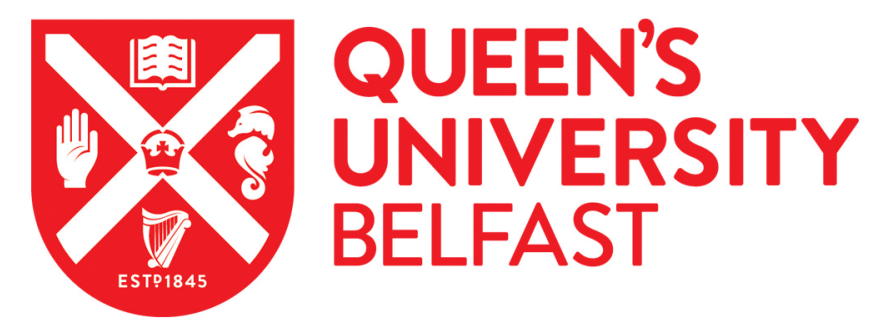

\title{
Spectral efficiency of multipair massive MIMO two-way relaying with imperfect CSI
}

Kong, C., Zhong, C., Matthaiou, M., Björnson, E., \& Zhang, Z. (2019). Spectral efficiency of multipair massive MIMO two-way relaying with imperfect CSI. IEEE Transactions on Vehicular Technology.

https://doi.org/10.1109/TVT.2019.2915005

\section{Published in:}

IEEE Transactions on Vehicular Technology

\section{Document Version:}

Peer reviewed version

Queen's University Belfast - Research Portal:

Link to publication record in Queen's University Belfast Research Portal

\section{Publisher rights}

(c) 2019 IEEE.

This work is made available online in accordance with the publisher's policies. Please refer to any applicable terms of use of the publisher.

\section{General rights}

Copyright for the publications made accessible via the Queen's University Belfast Research Portal is retained by the author(s) and / or other copyright owners and it is a condition of accessing these publications that users recognise and abide by the legal requirements associated with these rights.

Take down policy

The Research Portal is Queen's institutional repository that provides access to Queen's research output. Every effort has been made to ensure that content in the Research Portal does not infringe any person's rights, or applicable UK laws. If you discover content in the Research Portal that you believe breaches copyright or violates any law, please contact openaccess@qub.ac.uk. 


\title{
Spectral Efficiency of Multipair Massive MIMO Two-Way Relaying with Imperfect CSI
}

\author{
Chuili Kong, Student Member, IEEE, Caijun Zhong, Senior Member, IEEE, Michail Matthaiou, Senior Member, \\ IEEE, Emil Björnson, Senior Member, IEEE, and Zhaoyang Zhang, Member, IEEE
}

\begin{abstract}
We consider a two-way half-duplex relaying system where multiple pairs of single-antenna users exchange information assisted by a multiple-antenna relay. Taking into account the practical constraint of imperfect channel knowledge, we study the achievable sum spectral efficiency (SE) of the amplify-and-forward protocol, assuming that the relay employs maximum ratio processing. We derive a closed-form expression for the sum SE for arbitrary system parameters and a largescale approximation for the sum SE when the number of relay antennas, $M$, becomes sufficiently large. In addition, we study how the transmit power reduces with $M$ to maintain a desired SE. Our results show that by using a large number of relay antennas, the transmit powers of the user, relay, and pilot symbol can be scaled down proportionally to $1 / M^{\alpha}, 1 / M^{\beta}$, and $1 / M^{\gamma}$ for certain combinations of $\alpha, \beta$, and $\gamma$, respectively. This elegant power scaling law reveals a fundamental tradeoff between the transmit powers of the user/relay and pilot symbol. Finally, capitalizing on the new expressions for the sum SE, novel power allocation schemes are designed to further improve the sum SE.
\end{abstract}

Index Terms-Amplify-and-forward, geometric programming, massive MIMO, power scaling law, two-way relaying.

\section{INTRODUCTION}

Relaying is a low-complexity and cost-effective means to extend the network coverage and provide spatial diversity, which has attracted a great deal of research attention from both academia and industry [1]-[5]. Thus far, most practical

Manuscript received August 4, 2018, revised October 28, 2019 and January 15, 2019, accepted April 30, 2019. This work was supported in part by the National Natural Science Foundation of China (61671406, 61725104), NSFC-Zhejiang Joint Fund for the Integration of Industrialization and Informatization No. U1709219, and the Open Research Fund of State Key Laboratory of Integrated Services Networks under Grant ISN19-05. The work of M Matthaiou was supported by the RAEng/The Leverhulme Trust Senior Research Fellowship under Grant LTSRF1718 \14\2. The work of E. Björnson was supported by ELLIIT and CENIIT. The editor coordinating the review of this paper and approving it for publication was K. Adachi. (Corresponding author: Caijun Zhong)

C. Kong, C. Zhong and Z. Zhang are with the Zhejiang Provincial Key Laboratory of Information Processing, Communication and Networking, Institute of Information and Communication Engineering, Zhejiang University, Hangzhou 310027, China. C. Kong and C. Zhong are also with the State Key Laboratory of Integrated Services Networks, Xidian University, Xi'an 710126, China (e-mail: kcl_dut@163.com; caijunzhong@zju.edu.cn; sunrise.heaven@gmail.com).

M. Matthaiou is with the Institute of Electronics, Communications and Information Technology (ECIT), Queen's University Belfast, Belfast, BT3 9DT, U.K. (email: m.matthaiou@qub.ac.uk).

E. Björnson is with the Department of Electrical Engineering (ISY), Linköping University, Linköping, SE-581 83, Sweden (email: emil.bjornson@liu.se).

Part of this paper has been presented at the 2016 IEEE International Conference on Acoustics, Speech and Signal Processing (ICASSP), March 2016, Shanghai, China [15]. relaying systems are assumed to operate in the half-duplex mode where the relay does not transmit and receive signals simultaneously. Yet, such half-duplex mechanism incurs a $50 \%$ spectral efficiency (SE) loss. To reduce this loss in SE, two-way relaying was proposed in [6]-[12], where the two communicating nodes perform bidirectional simultaneous data transmission.

\section{A. Related Work}

Multipair two-way relaying is a sophisticated generalization of single-pair two-way relaying, where multiple pairs of users simultaneously establish a communication link with the aid of a single shared relay [13]-[17], hence substantially boosting the system SE. The major challenge is to properly handle the inter-pair interference from co-existing communication pairs. Thus far, a number of advanced techniques have been introduced to mitigate inter-pair interference, such as dirty-paper coding [18] and interference alignment [19]. Unfortunately, the practical implementation of these techniques is in general very complex. On the other hand, the massive multipleinput multiple-output (MIMO) paradigm has demonstrated superior interference suppression capabilities, with very simple and low-complexity linear processing [20]. Besides, the use of massive MIMO has the potential to achieve orders of magnitude spectral efficiency improvement with affordable signal processing complexity. Therefore, deploying large-scale antenna arrays in two-way relaying systems appears to be a very promising solution for inter-pair interference mitigation.

Some initial works have studied the fundamental performance of such systems [13], [14], [21]-[25]. In particular, for the half-duplex amplify-and-forward (AF) relaying, [13] investigated the achievable SE and power scaling laws of the maximum ratio (MR) and zero-forcing ( $\mathrm{ZF}$ ) processing schemes. Moreover, [14] derived a closed-form approximation of the SE of the MR scheme, and addressed the optimal user pair selection problem. Then, given minimum rate constraints, [21] proposed two algorithms to maximize the energy efficiency. For the full-duplex protocol, [22] studied the energy efficiency based on a practical power consumption model, while [23] considered antenna correlation and discovered the power scaling laws under various cases. However, one major limitation of the above works is that perfect channel state information (CSI) is assumed. Since obtaining perfect CSI is a formidable challenge in massive MIMO systems, it is important to look into the realistic scenario of imperfect CSI. An early attempt was made in [24], where the authors studied 
the sum SE performance of training-based systems utilizing the ZF scheme. Later, [25] investigated the asymptotic SE with MR and ZF, and designed an optimal power allocation strategy to maximize the sum SE under certain practical constraints. Although the derived results in [24], [25] are useful for understanding the impact of imperfect CSI on the system performance, a number of important questions remain to be addressed. For instance, the fundamental tradeoff between the transmit powers of pilot, user, and the relay remains an open problem. Our previous work of [26] has revealed this tradeoff for the decode-and-forward relaying system. Since the AF protocol does not need to decode the signals, it is preferred for applications which are sensitive to complexity and time latency, hence, is particularly suitable for the URLLC ecosystem. Therefore, providing a comprehensive analysis of multipair two-way relaying systems with the AF protocol is also of great interest. Motivated by this, we extend the analysis of [26] to the AF relaying systems, and present the achievable rate, the power tradeoff, and the power allocation design for the AF protocol. Our contributions are summarized as follows.

\section{B. Contributions}

- We investigate a multipair two-way AF relaying system that employs MR processing with imperfect CSI, and derive the SE expression in closed-form which is applicable for arbitrary system configurations and a large-scale approximation of the SE when $M \rightarrow \infty$, where $M$ is the number of relay antennas.

- We consider three different transmit powers, i.e., the relay's transmit power, the user's transmit power, and the transmit power of each pilot symbol, and characterize the interplay between them, which permits great flexibility in the design of practical systems. In contrast, most of the previous works only consider the transmit powers of the user and the relay, and ignore the power-scaling analysis in the channel training stage.

- We study the power allocation problem that maximizes the sum SE, subject to a total power constraint. Using the same method as in [25], a local optimum solution is obtained by solving a sequence of geometric programming (GP) problems. In addition, a closed-form power allocation strategy is obtained for the special case where users are independent and identically distributed, which suggests that half of the total power should be allocated to the relay while the rest is equally allocated to the users.

\section{Paper Outline and Notations}

The remainder of the paper is organized as follows: Section II introduces the considered multipair two-way half-duplex relaying system model. Section III presents the SE in closedform for arbitrary $M$ and a large-scale approximation when $M \rightarrow \infty$, with imperfect CSI, while Section IV studies the power scaling laws of different system configurations. The power allocation problem is discussed in Section V. The numerical results are verified in Section VI. Finally, Section VII provides some concluding remarks.
Notation: We use bold upper case letters to denote matrices, bold lower case letters to denote vectors and lower case letters to denote scalars. Moreover, $(\cdot)^{H},(\cdot)^{*},(\cdot)^{T}$, and $(\cdot)^{-1}$ represent the conjugate transpose operator, the conjugate operator, the transpose operator, and the matrix inverse, respectively. Also, $\|\cdot\|$ is the Euclidian norm, $\|\cdot\|_{\mathrm{F}}$ denotes the Frobenius norm, and $|\cdot|$ is the absolute value. In addition, $\mathbf{x} \sim \mathcal{C N}(\mathbf{0}, \boldsymbol{\Sigma})$ denotes a circularly symmetric complex Gaussian random vector $\mathbf{x}$ with covariance matrix $\boldsymbol{\Sigma}, \mathbf{I}_{k}$ is the identity matrix of size $k$, while 1 denotes the vector whose elements are all 1. Finally, the statistical expectation operator is represented by $\mathrm{E}\{\cdot\}$, the variance operator is $\operatorname{Var}(\cdot)$, and the trace is denoted by $\operatorname{tr}(\cdot)$.

\section{System Model}

We consider a multipair two-way relaying communication network consisting of a relay $\mathrm{T}_{R}$ with $M$ antennas, and $N$ pairs of single-antenna users $\mathrm{T}_{A, i}$ and $\mathrm{T}_{B, i}, i=1, \ldots, N$. We assume that the direct links between $\mathrm{T}_{A, i}$ and $\mathrm{T}_{B, i}$ do not exist due to large obstacles or severe shadowing. Thus, they exchange information with each other via $\mathrm{T}_{R}$, as shown in Fig. 1. The relay operates in the half-duplex mode, i.e., it cannot transmit and receive simultaneously.



Fig. 1: Illustration of the multipair two-way relaying system.

We assumed that the system operates under the timedivision duplex protocol and channel reciprocity holds. The channels from $\mathrm{T}_{A, i}$ to $\mathrm{T}_{R}$, and $\mathrm{T}_{B, i}$ to $\mathrm{T}_{R}$ can be respectively denoted as $\mathbf{G}_{A R} \triangleq\left[\mathbf{g}_{A R, 1}, \ldots, \mathbf{g}_{A R, N}\right] \in \mathbb{C}^{M \times N}$ and $\mathbf{G}_{R B} \triangleq\left[\mathbf{g}_{R B, 1}, \ldots, \mathbf{g}_{R B, N}\right] \in \mathbb{C}^{M \times N}$. More precisely, by accounting for both small-scale and large-scale fading, the channel vectors can be expressed as $\mathbf{g}_{A R, i} \sim \mathcal{C N}\left(\mathbf{0}, \beta_{A R, i} \mathbf{I}_{M}\right)$ and $\mathbf{g}_{R B, i} \sim \mathcal{C N}\left(\mathbf{0}, \beta_{R B, i} \mathbf{I}_{M}\right)$, where $\beta_{A R, i}$ and $\beta_{R B, i}$ model the large-scale path-loss and shadowing effects that are assumed to be constant over many coherence intervals and known a priori.

\section{A. Channel Training Stage}

In the channel training stage, the channels $\mathbf{G}_{A R}$ and $\mathbf{G}_{R B}$ are estimated by utilizing uplink pilots [20]. In each coherence interval of length $\tau_{c}, \mathrm{~T}_{A, i}$ and $\mathrm{T}_{B, i}$ simultaneously transmit their mutually orthogonal pilot sequences of length $\tau_{p}$ to $\mathrm{T}_{R}$, 
for $i=1, \ldots, N$. Thus, the received pilot matrix at the relay is given by

$$
\mathbf{Y}_{p}=\sqrt{\tau_{p} p_{p}} \mathbf{G}_{A R} \boldsymbol{\Phi}_{A}^{T}+\sqrt{\tau_{p} p_{p}} \mathbf{G}_{R B} \boldsymbol{\Phi}_{B}^{T}+\mathbf{N}_{p}
$$

where $p_{p}$ is the transmit power of each pilot symbol, $\mathbf{N}_{p}$ is AWGN matrix including i.i.d. $\mathcal{C N}(0,1)$ elements. ${ }^{1}$ The matrices $\boldsymbol{\Phi}_{A} \in \mathbb{C}^{\tau_{p} \times N}$ and $\boldsymbol{\Phi}_{B} \in \mathbb{C}^{\tau_{p} \times N}$ are respectively the pilot sequences transmitted from $\mathrm{T}_{A, i}$ and $\mathrm{T}_{B, i}$, and satisfy $\tau_{p} \geq 2 N, \boldsymbol{\Phi}_{A}^{T} \boldsymbol{\Phi}_{A}^{*}=\mathbf{I}_{N}, \boldsymbol{\Phi}_{B}^{T} \boldsymbol{\Phi}_{B}^{*}=\mathbf{I}_{N}$, and $\boldsymbol{\Phi}_{A}^{T} \boldsymbol{\Phi}_{B}^{*}=\mathbf{0}_{N}$ to preserve orthogonality of the pilots.

As in [1], [24], [27], we assume that $\mathrm{T}_{R}$ uses the minimum mean-square-error (MMSE) estimator to estimate the channels $\mathbf{G}_{A R}$ and $\mathbf{G}_{R B}$. From the property of MMSE channel estimation, the channel vectors can be decomposed as

$$
\begin{aligned}
& \mathbf{g}_{A R, i}=\hat{\mathbf{g}}_{A R, i}+\mathbf{e}_{A R, i}, \\
& \mathbf{g}_{R B, i}=\hat{\mathbf{g}}_{R B, i}+\mathbf{e}_{R B, i},
\end{aligned}
$$

where $\hat{\mathbf{g}}_{A R, i}, \hat{\mathbf{g}}_{R B, i}, \mathbf{e}_{A R, i}$, and $\mathbf{e}_{R B, i}$ are the $i$-th columns of the estimated matrices $\hat{\mathbf{G}}_{A R}, \hat{\mathbf{G}}_{R B}$, and the estimation error matrices $\mathbf{E}_{A R}$ and $\mathbf{E}_{R B}$, respectively, which are mutually independent. Furthermore, the elements of $\hat{\mathbf{g}}_{A R, i}, \mathbf{e}_{A R, i}$ are Gaussian random variables with zero mean, variance $\sigma_{A R, i}^{2}$ and $\tilde{\sigma}_{A R, i}^{2}$, respectively, where $\sigma_{A R, i}^{2} \triangleq \frac{\tau_{p} p_{p} \beta_{A R, i}^{2}}{1+\tau_{p} p_{p} \beta_{A R, i}}$ and $\tilde{\sigma}_{A R, i}^{2} \triangleq \frac{\beta_{A R, i}}{1+\tau_{p} p_{p} \beta_{A R, i}}$. Similarly, the elements of $\hat{\mathbf{g}}_{R B, i}$, and $\mathbf{e}_{R B, i}$ are complex Gaussian random variables with zero mean, variance $\sigma_{R B, i}^{2}$ and $\tilde{\sigma}_{R B, i}^{2}$, respectively, where $\sigma_{R B, i}^{2} \triangleq$ $\frac{\tau_{p} p_{p} \beta_{R B, i}^{2}}{1+\tau_{p} p_{p} \beta_{R B, i}}$ and $\tilde{\sigma}_{R B, i}^{2} \triangleq \frac{\beta_{R B, i}}{1+\tau_{p} p_{p} \beta_{R B, i}}$.

\section{B. Data Transmission Stage}

The data transmission stage consists of two separate phases. In the first phase, the $N$ user pairs simultaneously transmit their respective signals to $\mathrm{T}_{R}$. Thus, the received signal at $\mathrm{T}_{R}$ is given by

$$
\mathbf{y}_{r}=\sum_{i=1}^{N}\left(\sqrt{p_{A, i}} \mathbf{g}_{A R, i} x_{A, i}+\sqrt{p_{B, i}} \mathbf{g}_{R B, i} x_{B, i}\right)+\mathbf{n}_{R}
$$

where $x_{A, i}$ and $x_{B, i}$ are complex Gaussian signals with zero mean and unit power transmitted by the $i$-th user pair, $p_{A, i}$ and $p_{B, i}$ are the average transmit power of $\mathrm{T}_{A, i}$ and $\mathrm{T}_{B, i}$, respectively, and $\mathbf{n}_{R}$ is a vector of additive white Gaussian noise (AWGN) at $\mathrm{T}_{R}$, whose elements are identically and independently distributed (i.i.d.) $\mathcal{C N}(0,1)$.

In the second phase, the relay first applies MR processing on the received signal ${ }^{2}$, and then broadcasts it to the users. Thus, the transmit signal from $\mathrm{T}_{R}$ can be written as

$$
\mathbf{y}_{t}=\rho \mathbf{F} \mathbf{y}_{r},
$$

where the processing matrix $\mathbf{F} \in \mathbb{C}^{M \times M}$ is given by [14]

$$
\mathbf{F}=\mathbf{B}^{*} \mathbf{A}^{H}
$$

${ }^{1}$ Note that for notational convenience, we set the noise variance to be 1 throughout the paper. With this convention, the transmit power in the paper can be interpreted as the normalized transmit signal to noise (expressed in $\mathrm{dB})$.

${ }^{2}$ Note that MR is a very attractive linear processing technique in the context of massive MIMO systems due to its low complexity. Most importantly, it can be implemented in a distributed manner [20]. with $\mathbf{A} \triangleq\left[\hat{\mathbf{G}}_{A R}, \hat{\mathbf{G}}_{R B}\right]$, and $\mathbf{B} \triangleq\left[\hat{\mathbf{G}}_{R B}, \hat{\mathbf{G}}_{A R}\right]$, and $\rho$ is chosen to satisfy the long-term total transmit power constraint at the relay, namely, $\mathrm{E}\left\{\left\|\mathbf{y}_{t}\right\|^{2}\right\}=p_{r}$, where $p_{r}$ is the average transmit power of the relay. Thus, we have $\rho$, which is given by (7), shown on the top of the next page.

As a result, the received signals at $\mathrm{T}_{A, i}$ and $\mathrm{T}_{B, i}$ are respectively given by

$$
\begin{aligned}
& z_{A, i}=\mathbf{g}_{A R, i}^{T} \mathbf{y}_{t}+n_{A, i}, \\
& z_{B, i}=\mathbf{g}_{R B, i}^{T} \mathbf{y}_{t}+n_{B, i},
\end{aligned}
$$

where $n_{A, i} \sim \mathcal{C N}(0,1)$ and $n_{B, i} \sim \mathcal{C N}(0,1)$ represent the AWGN at $\mathrm{T}_{A, i}$ and $\mathrm{T}_{B, i}$.

\section{SPECTRAL EFFICIENCY}

In this section, we derive the SE expression in closed-form for MR processing, which is applicable for arbitrary number of relay antennas. Furthermore, a large-scale approximation of the SE is deduced when antenna arrays at the relay are very large.

Without loss of generality, we focus on the characterization of the achievable SE of user $\mathrm{T}_{A, i}$. When $\mathrm{T}_{A, i}$ receives the superimposed signal from $\mathrm{T}_{R}$, it first attempts to subtract its own transmitted message according to its available CSI (known as self-interference cancellation). Here, we consider the realistic case where the users only have the statistical CSI that is obtained by the feedback from the relay. Therefore, after subtracting the partial self-interference term $\rho \sqrt{p_{A, i} \mathrm{E}}\left\{\mathbf{g}_{A R, i}^{\mathrm{T}} \mathbf{F} \mathbf{g}_{A R, i}\right\} x_{A, i}$, the received signal at $\mathrm{T}_{A, i}$ is re-expressed as

$$
\begin{aligned}
\hat{z}_{A, i} & =z_{A, i}-\rho \sqrt{p_{A, i}} \mathbf{E}\left\{\mathbf{g}_{A R, i}^{\mathrm{T}} \mathbf{F} \mathbf{g}_{A R, i}\right\} x_{A, i} \\
& =\underbrace{\rho \sqrt{p_{B, i}} \mathbf{E}\left\{\mathbf{g}_{A R, i}^{\mathrm{T}} \mathbf{F g}_{R B, i}\right\} x_{B, i}}_{\text {desired signal }} \\
& +\underbrace{\rho \sqrt{p_{B, i}}\left(\mathbf{g}_{A R, i}^{\mathrm{T}} \mathbf{F} \mathbf{g}_{R B, i}-\mathbf{E}\left\{\mathbf{g}_{A R, i}^{\mathrm{T}} \mathbf{F} \mathbf{g}_{R B, i}\right\}\right) x_{B, i}}_{\text {gain uncertainty }} \\
& +\underbrace{\rho \sqrt{p_{A, i}}\left(\mathbf{g}_{A R, i}^{\mathrm{T}} \mathbf{F} \mathbf{g}_{A R, i}-\mathrm{E}\left\{\mathbf{g}_{A R, i}^{\mathrm{T}} \mathbf{F} \mathbf{g}_{A R, i}\right\}\right) x_{A, i}}_{\text {residual self-interference }} \\
& +\underbrace{\rho \sum_{j \neq i} \mathbf{g}_{A R, i}^{\mathrm{T}} \mathbf{F}\left(\sqrt{p_{A, i}} \mathbf{g}_{A R, j} x_{A, j}+\sqrt{p_{B, i}} \mathbf{g}_{R B, j} x_{B, j}\right)}_{\text {inter-user interference }} \\
& +\underbrace{\rho \mathbf{g}_{A R, i}^{\mathrm{T}} \mathbf{F} \mathbf{n}_{R}+n_{A, i}}_{\text {compound noise }} .
\end{aligned}
$$

Using a standard approach as in [1], [29], an ergodic achievable $\mathrm{SE}$ of $\mathrm{T}_{A, i}$ is

$$
R_{A, i}=\frac{\tau_{c}-\tau_{p}}{2 \tau_{c}} \log _{2}\left(1+\frac{A_{i}}{B_{i}+C_{i}+D_{i}+E_{i}}\right),
$$

where the pre-log factor $1 / 2$ is introduced for the half-duplex 


$$
\rho=\sqrt{\frac{p_{r}}{\sum_{i=1}^{N}\left(p_{A, i} \mathrm{E}\left\{\left\|\mathbf{F g}_{A R, i}\right\|^{2}\right\}+p_{B, i} \mathrm{E}\left\{\left\|\mathbf{F g}_{R B, i}\right\|^{2}\right\}\right)+\mathrm{E}\left\{\|\mathbf{F}\|_{\mathrm{F}}^{2}\right\}}} .
$$

mode, and

$$
\begin{aligned}
A_{i} & =p_{B, i}\left|\mathrm{E}\left\{\mathbf{g}_{A R, i}^{\mathrm{T}} \mathbf{F} \mathbf{g}_{R B, i}\right\}\right|^{2}, \\
B_{i} & =p_{B, i} \operatorname{Var}\left(\mathbf{g}_{A R, i}^{\mathrm{T}} \mathbf{F} \mathbf{g}_{R B, i}\right), \\
C_{i} & =p_{A, i} \operatorname{Var}\left(\mathbf{g}_{A R, i}^{\mathrm{T}} \mathbf{F} \mathbf{g}_{A R, i}\right), \\
D_{i} & =\sum_{j \neq i} \mathrm{E}\left\{p_{A, i}\left|\mathbf{g}_{A R, i}^{\mathrm{T}} \mathbf{F} \mathbf{g}_{A R, j}\right|^{2}\right\} \\
& +\sum_{j \neq i} \mathrm{E}\left\{p_{B, i}\left|\mathbf{g}_{A R, i}^{\mathrm{T}} \mathbf{F} \mathbf{g}_{R B, j}\right|^{2}\right\}, \\
E_{i} & =\mathrm{E}\left\{\left\|\mathbf{g}_{A R, i}^{\mathrm{T}} \mathbf{F}\right\|^{2}\right\}+\frac{1}{\rho^{2}} .
\end{aligned}
$$

Thus, the ergodic sum SE of the multipair two-way AF relaying system is given by

$$
R=\sum_{i=1}^{N}\left(R_{A, i}+R_{B, i}\right),
$$

where $R_{B, i}$ is the $\mathrm{SE}$ of $\mathrm{T}_{B, i}$, which can be derived in a similar fashion.

Theorem 1: With the AF protocol, the ergodic SE for an arbitrary number of relay antennas is given by (11) with

$$
\begin{aligned}
& A_{i}=p_{B, i} M^{2}(M+1)^{2} \sigma_{A R, i}^{4} \sigma_{R B, i}^{4} \\
& B_{i}=p_{B, i} 2 M(M+1) \beta_{A R, i} \beta_{R B, i} \sum_{n \neq i} \sigma_{A R, n}^{2} \sigma_{R B, n}^{2} \\
& +p_{B, i} M(M+1) \sigma_{A R, i}^{2} \sigma_{R B, i}^{2}\left(\beta_{A R, i} \sigma_{R B, i}^{2}+\beta_{R B, i} \sigma_{A R, i}^{2}\right) \\
& +p_{B, i} 2 M(M+1) \sigma_{A R, i}^{2} \sigma_{R B, i}^{2} \tilde{\sigma}_{A R, i}^{2} \tilde{\sigma}_{R B, i}^{2} \\
& +p_{B, i} 2 M(M+1)^{2} \sigma_{A R, i}^{4} \sigma_{R B, i}^{4} \\
& +p_{B, i} M(M+1)(M+2) \sigma_{A R, i}^{2} \tilde{\sigma}_{A R, i}^{2} \sigma_{R B, i}^{2}\left(\sigma_{A R, i}^{2}+\sigma_{R B, i}^{2}\right) \\
& C_{i}=4 p_{A, i} M(M+1) \beta_{A R, i}^{2} \sum_{n \neq i} \sigma_{A R, n}^{2} \sigma_{R B, n}^{2} \\
& +4 p_{A, i} \sigma_{A R, i}^{2} \sigma_{R B, i}^{2} M(M+1)(M+2) \sigma_{A R, i}^{4} \\
& +4 p_{A, i} \sigma_{A R, i}^{2} \sigma_{R B, i}^{2} M(M+1)(M+5) \sigma_{A R, i}^{2} \tilde{\sigma}_{A R, i}^{2} \\
& +4 p_{A, i} \sigma_{A R, i}^{2} \sigma_{R B, i}^{2} M(M+1) \tilde{\sigma}_{A R, i}^{4} \\
& D_{i}=\sum_{j \neq i} 2 M(M+1) \beta_{A R, i} t_{j} \sum_{n \neq i, j} \sigma_{A R, n}^{2} \sigma_{R B, n}^{2} \\
& +\sum_{j \neq i} M \sigma_{A R, i}^{2} \sigma_{R B, i}^{2} t_{j} m \sigma_{A R, i}^{2} \\
& +\sum_{j \neq i} M \sigma_{A R, i}^{2} \sigma_{R B, i}^{2} t_{j} 2(M+1) \tilde{\sigma}_{A R, i}^{2} \\
& +\sum_{j \neq i} M \beta_{A R, i} \sigma_{A R, j}^{2} \sigma_{R B, j}^{2} m\left(p_{A, i} \sigma_{A R, j}^{2}+p_{B, i} \sigma_{R B, j}^{2}\right) \\
& +\sum_{j \neq i} 2(M+1)\left(p_{A, i} \tilde{\sigma}_{A R, j}^{2}+p_{B, i} \tilde{\sigma}_{R B, j}^{2}\right) \\
& +j
\end{aligned}
$$

$$
\begin{aligned}
& E_{i}=2 M(M+1) \beta_{A R, i} \sum_{n \neq i} \sigma_{A R, n}^{2} \sigma_{R B, n}^{2} \\
& +M \sigma_{A R, i}^{2} \sigma_{R B, i}^{2}\left(m \sigma_{A R, i}^{2}+2(M+1) \tilde{\sigma}_{A R, i}^{2}\right) \\
& +\frac{1}{p_{r}} \sum_{i=1}^{N} M \sigma_{A R, i}^{2} \sigma_{R B, i}^{2} m\left(\sigma_{A R, i}^{2} p_{A, i}+\sigma_{R B, i}^{2} p_{B, i}\right) \\
& +\frac{1}{p_{r}} \sum_{i=1}^{N} M \sigma_{A R, i}^{2} \sigma_{R B, i}^{2} 2(M+1)\left(\tilde{\sigma}_{A R, i}^{2} p_{A, i}+\tilde{\sigma}_{R B, i}^{2} p_{B, i}\right) \\
& +\frac{1}{p_{r}} \sum_{i=1}^{N} 2 M(M+1) t_{i} \sum_{n \neq i} \sigma_{A R, n}^{2} \sigma_{R B, n}^{2} \\
& +\frac{1}{p_{r}} 2 M(M+1) \sum_{n=1}^{N} \sigma_{A R, n}^{2} \cdot \sigma_{R B, n}^{2},
\end{aligned}
$$

where $t_{j}=p_{A, i} \beta_{A R, j}+p_{B, i} \beta_{R B, j} \quad$ and $m=$ $(M+1)(M+3)$.

Proof: See Appendix A.

Theorem 1 presents the closed-form SE, which is applicable to arbitrary number of relay antennas. While Theorem 1 enables efficient evaluation of the SE, it is in general difficult to extract insights due to the involved expression. As such, we now look into the asymptotic regime where the relay has a large number of antennas, and derive a simple and accurate approximation for the SE.

Theorem 2: With the AF protocol, as the number of relay antennas grows to infinity, then we have $R_{A, i}-\tilde{R}_{A, i} \stackrel{M \rightarrow \infty}{\longrightarrow} 0$, where $\tilde{R}_{A, i}$ is given by

$$
\tilde{R}_{A, i} \triangleq \frac{\tau_{c}-\tau_{p}}{2 \tau_{c}} \log _{2}\left(1+\frac{p_{B, i} M}{\tilde{B}_{i}+\tilde{C}_{i}+\tilde{D}_{i}+\tilde{E}_{i}}\right),
$$

where

$\tilde{B}_{i} \triangleq p_{B, i}\left(\frac{\beta_{R B, i}}{\sigma_{R B, i}^{2}}+\frac{\beta_{A R, i}}{\sigma_{A R, i}^{2}}\right)$,

$\tilde{C}_{i} \triangleq \frac{4 p_{A, i} \beta_{A R, i}}{\sigma_{R B, i}^{2}}$,

$\tilde{D}_{i} \triangleq \sum_{j \neq i} p_{A, j}\left(\frac{\beta_{A R, j}}{\sigma_{R B, i}^{2}}+\frac{\sigma_{A R, j}^{4} \sigma_{R B, j}^{2} \beta_{A R, i}}{\sigma_{A R, i}^{4} \sigma_{R B, i}^{4}}\right)$

$+\sum_{j \neq i} p_{B, j}\left(\frac{\beta_{R B, j}}{\sigma_{R B, i}^{2}}+\frac{\sigma_{A R, j}^{2} \sigma_{R B, j}^{4} \beta_{A R, i}}{\sigma_{A R, i}^{4} \sigma_{R B, i}^{4}}\right)$,

$\tilde{E}_{i} \triangleq \frac{1}{\sigma_{R B, i}^{2}}$

$+\frac{1}{p_{r} \sigma_{A R, i}^{4} \sigma_{R B, i}^{4}} \sum_{n=1}^{N} \sigma_{A R, n}^{2} \sigma_{R B, n}^{2}\left(p_{A, n} \sigma_{A R, n}^{2}+p_{B, n} \sigma_{R B, n}^{2}\right)$.

Theorem 2 presents a large-scale approximation of the $i$-th user's SE which is asymptotically tight. Despite being obtained under the massive array assumption, the approximation turns 
out to be very accurate even for practical number of relay antennas. Most importantly, it is easy to observe the impact of various factors on the asymptotic SE. It can be seen that the individual user SE $\tilde{R}_{A, i}$ decreases with the number of user pairs $N$; this is anticipated since a higher number of users increases the amount of inter-user interference. Now, we focus on studying the impact of the transmit power of $i$-th user pair $p_{A, i}$ and $p_{B, i}$, the transmit power of the relay $p_{r}$, and the transmit power of each pilot symbol $p_{p}$ on the system performance. As can be seen, when $p_{A, i} \rightarrow \infty$ and $p_{B, i} \rightarrow \infty$, the SE is limited by $p_{r}$ and $p_{p}$; in contrast, it is limited by $p_{A, i}, p_{B, i}$, and $p_{p}$ when $p_{r} \rightarrow \infty$.

\section{Power Scaling Laws}

In this section, we present a detailed analysis of the power scaling laws. In other words, we characterize that how the powers can be reduced proportionally to $M$ while maintaining a desired non-zero SE. Note that power actually refers to the signal-to-noise ratio and thus the analysis shows how to achieve the SE either using less power or extending the coverage. Since our main focus is on the extent of power reduction rather than the behavior of a particular user, we assume that all the users have the same transmit power, i.e., $p_{A, i}=p_{B, i}=p_{u}$. Then, based on three different scenarios, we characterize the interplay between the relay's transmit power $p_{r}$, the transmit power of each user $p_{u}$, and the transmit power of each pilot symbol $p_{p}$, as the number of relay antennas $M$ grows to infinity.

\section{A. Scenario A}

In scenario $\mathrm{A}, p_{u}$ and $p_{r}$ are fixed, while $p_{p}=\frac{E_{p}}{M^{\gamma}}$ with $\gamma>0$, and $E_{p}$ being a constant. Such a scenario represents the potential of power saving in the training stage.

Now define the Condition I: $E_{p} \ll \tau_{p} M^{\gamma} \times$ $\min \left\{\beta_{A R, i}, \beta_{R B, i}\right\}$, then we have the following important result:

Theorem 3: For fixed and finite $p_{u}, p_{r}$ and $E_{p}$, when $p_{p}=$ $\frac{E_{p}}{M^{\gamma}}$ with $\gamma>0$, as $M \rightarrow \infty$ and Condition I holds, we have

$$
R_{A, i}-\frac{\tau_{c}-\tau_{p}}{2 \tau_{c}} \log _{2}\left(1+\frac{\tau_{p} E_{p} M^{1-\gamma}}{\hat{B}_{i}+\hat{C}_{i}+\hat{D}_{i}+\hat{E}_{i}}\right) \stackrel{M \rightarrow \infty}{\longrightarrow} 0,
$$

where

$$
\begin{aligned}
& \hat{B}_{i} \triangleq \frac{1}{\beta_{R B, i}}+\frac{1}{\beta_{A R, i}} \\
& \hat{C}_{i} \triangleq \frac{4 \beta_{A R, i}}{\beta_{R B, i}^{2}} \\
& \hat{D}_{i} \triangleq \sum_{j \neq i}\left(\frac{\beta_{A R, j}+\beta_{R B, j}}{\beta_{R B, i}^{2}}+\frac{\beta_{A R, j}^{4} \beta_{R B, j}^{2}+\beta_{A R, j}^{2} \beta_{R B, j}^{4}}{\beta_{A R, i}^{3} \beta_{R B, i}^{4}}\right) \\
& \hat{E}_{i} \triangleq \frac{1}{p_{u} \beta_{R B, i}^{2}} \\
& +\frac{1}{p_{r} \beta_{A R, i}^{4} \beta_{R B, i}^{4}} \sum_{n=1}^{N} \beta_{A R, n}^{2} \beta_{R B, n}^{2}\left(\beta_{A R, n}^{2}+\beta_{R B, n}^{2}\right)
\end{aligned}
$$

Theorem 3 implies that the large-scale approximation of the SE $R_{A, i}$ depends on the choice of $\gamma$. When $\gamma>1, R_{A, i}$ reduces to zero due to the poor channel estimation accuracy caused by over-reducing the pilot transmit power. In contrast, when $0<\gamma<1, R_{A, i}$ grows without bound, which indicates that the transmit power of each pilot symbol can be scaled down arbitrarily within this regime. Finally, when $\gamma=1, R_{A, i}$ converges to a non-zero limit, which suggests that with large antenna arrays, the transmit power of each pilot symbol can be scaled down at most by $1 / M$ to maintain a given SE.

\section{B. Scenario B}

In scenario $\mathrm{B}, p_{p}$ is fixed, while $p_{u}=\frac{E_{u}}{M^{\alpha}}, p_{r}=\frac{E_{r}}{M^{\beta}}$, with $\alpha \geq 0$ and $\beta \geq 0$, and $E_{u}, E_{r}$ are constants. Hence, the channel estimation accuracy remains unchanged, and the objective is to study the potential power savings in the data transmission stage, as well as, the interplay between the user and relay transmit powers.

Now define the following conditions, namely, Condition II: $\frac{M^{\alpha}}{E_{u} \sigma_{R B, i}^{2}}+\frac{M^{\beta}}{E_{r} \sigma_{A R, i}^{4} \sigma_{R B, i}^{4}} \sum_{n=1}^{N} \sigma_{A R, n}^{2} \sigma_{R B, n}^{2}\left(\sigma_{A R, n}^{2}+\sigma_{R B, n}^{2}\right) \gg$ $\frac{\beta_{R B, i}}{\sigma_{R B, i}^{2}}+\frac{\beta_{A R, i}}{\sigma_{A R, i}^{2}}+\frac{4 \beta_{A R, i}}{\sigma_{R B, i}^{2}}+\sum_{j \neq i}\left(\frac{\beta_{A R, j}}{\sigma_{R B, i}^{2}}+\frac{\sigma_{A R, j}^{4} \sigma_{R B, j}^{2} \beta_{A R, i}}{\sigma_{A R, i}^{4} \sigma_{R B, i}^{4}}\right)+$ $\sum_{j \neq i}\left(\frac{\beta_{R B, j}}{\sigma_{R B, i}^{2}}+\frac{\sigma_{A R, j}^{2} \sigma_{R B, j}^{4} \beta_{A R, i}}{\sigma_{A R, i}^{4} \sigma_{R B, i}^{4}}\right)$, Condition III:

$\frac{1}{E_{u}} \gg \frac{M^{\beta-1}}{E_{r} \sigma_{A R, i}^{4} \sigma_{R B, i}^{2}} \sum_{n=1}^{N} \sigma_{A R, n}^{2} \sigma_{R B, n}^{2}\left(\sigma_{A R, n}^{2}+\sigma_{R B, n}^{2}\right)$, and Condition IV:

$\frac{M^{\alpha-1}}{E_{u}} \ll \frac{1}{E_{r} \sigma_{A R, i}^{4} \sigma_{R B, i}^{2}} \sum_{n=1}^{N} \sigma_{A R, n}^{2} \sigma_{R B, n}^{2}\left(\sigma_{A R, n}^{2}+\sigma_{R B, n}^{2}\right)$, then we have:

Theorem 4: For fixed and finite $p_{p}, E_{u}$, and $E_{r}$, when $p_{u}=$ $\frac{E_{u}}{M^{\alpha}}, p_{r}=\frac{E_{r}}{M^{\beta}}$, with $\alpha \geq 0, \beta \geq 0$, as $M \rightarrow \infty$ and Condition II holds, we have (33), which is shown on the top of the next page.

Theorem 4 reveals that in Scenario B, the estimation error, the residual self-interference, and the inter-user interference vanish completely, and only the compound noise remains, as $M \rightarrow \infty$. The reason is that the power scaling pushes the system into a noise limited regime. Moreover, it is observed that the compound noise consists of two parts, namely Part I and Part II as shown in (33), which represent the noise at the relay and the noise at the user $\mathrm{T}_{A, i}$, respectively. This observation can be interpreted as: when both the transmit powers of each user and the relay are scaled down inversely proportional to $M$, the effect of noise becomes increasingly significant. In addition, we can also see that when the channel estimation accuracy is fixed, the large-scale approximation of the SE $R_{A, i}$ depends on the value of $\alpha$ and $\beta$. When we cut down the transmit powers of the relay and/or of each user too much, namely, $\alpha>1$ and/or $\beta>1, R_{A, i}$ converges to zero. On the other hand, $R_{A, i}$ grows unboundedly for $0 \leq \alpha<1$ and $0 \leq \beta<1$. Only if $\alpha=1$ and/or $\beta=1, R_{A, i}$ converges to a finite limit as detailed in the following corollaries.

Corollary 1: For fixed and finite $p_{p}, E_{u}$, and $E_{r}$, when $\alpha=\beta=1$, namely, $p_{u}=\frac{E_{u}}{M}, p_{r}=\frac{E_{r}}{M}$, as $M \rightarrow \infty$ and Condition II holds, the SE has the limit (34), which is shown on the top of the next page. 


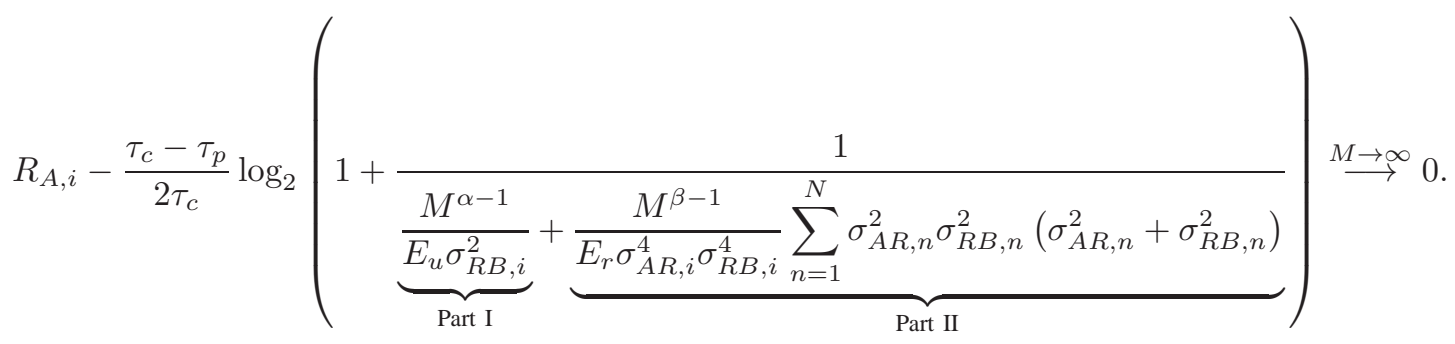

$$
R_{A, i} \rightarrow \frac{\tau_{c}-\tau_{p}}{2 \tau_{c}} \log _{2}\left(1+\frac{1}{\frac{1}{E_{u} \sigma_{R B, i}^{2}}+\frac{1}{E_{r} \sigma_{A R, i}^{4} \sigma_{R B, i}^{4}} \sum_{n=1}^{N}\left(\sigma_{A R, n}^{2} \sigma_{R B, n}^{2}\left(\sigma_{A R, n}^{2}+\sigma_{R B, n}^{2}\right)\right)}\right)
$$

From Corollary 1, we observe that when both the transmit powers of the relay and of each user are scaled down with the same speed, i.e., $1 / M, R_{A, i}$ converges to a non-zero limit. Moreover, this non-zero limit increases with $E_{u}$ and $E_{r}$, as expected. Now consider the special case of all the links having the same large-scale fading, e.g., $\beta_{A R, i}=\beta_{R B, i}=1$, for $i=1, \ldots, N$, then the sum SE of the system reduces to

$$
R \rightarrow \frac{\tau_{c}-\tau_{p}}{\tau_{c}} N \log _{2}\left(1+\frac{\sigma_{1}^{2} E_{u} E_{r}}{E_{r}+2 N E_{u}}\right),
$$

where $\sigma_{1}^{2}=\frac{\tau_{p} p_{p}}{\tau_{p} p_{p}+1}$. Therefore, the sum SE in (35) is equal to the one of $N$ parallel single-input single-output channels with transmit power $\frac{\sigma_{1}^{2} E_{u} E_{r}}{E_{r}+2 N E_{u}}$, without interference and small-scale fading. Note that we only need $\frac{2 N\left(E_{u}+\tau_{p} E_{p}\right)+E_{r}}{M}$ amount of power (the transmit power of each user is $\frac{E_{u}}{M}$, the transmit power of each pilot sequence is $\frac{\tau_{p} E_{p}}{M}$, and the transmit power of the relay is $\frac{E_{r}}{M}$ ) in multipair two-way AF relaying systems when using very large number of relay antennas. This represents a remarkable power reduction, thereby showcasing the huge benefits from the perspective of radiated energy efficiency by deploying large antenna arrays.

Corollary 2: For fixed and finite $p_{p}, E_{u}$ and $E_{r}$, when $\alpha=$ 1 and $0 \leq \beta<1$, namely, $p_{u}=\frac{E_{u}}{M}, p_{r}=\frac{E_{r}}{M^{\beta}}$, as $M \rightarrow \infty$ and Conditions II and III hold, the SE has the limit

$$
R_{A, i} \rightarrow \frac{\tau_{c}-\tau_{p}}{2 \tau_{c}} \log _{2}\left(1+E_{u} \sigma_{R B, i}^{2}\right) .
$$

Corollary 2 presents an interesting phenomenon, that if the transmit power of each user is overly cut down compared to the reduction of the relay transmit power, the value of Part I is dominant compared to Part II in (33), and thus $R_{A, i}$ converges to a non-zero limit that is determined by the noise at the relay. This observation is intuitive, since when both $\mathrm{T}_{A, i}$ and $\mathrm{T}_{B, i}$ transmit with extremely low power, the effect of noise at the relay becomes the performance limiting factor. Similarly, when $0 \leq \alpha<\beta=1$, we have the following corollary.

Corollary 3: For fixed and finite $p_{p}, E_{u}$, and $E_{r}$, when $0 \leq \alpha<1$ and $\beta=1$, namely, $p_{u}=\frac{E_{u}}{M^{\alpha}}, p_{r}=\frac{E_{r}}{M}$, as
$M \rightarrow \infty$ and Conditions II and IV hold, the SE has the limit

$$
\begin{aligned}
& R_{A, i} \rightarrow \frac{\tau_{c}-\tau_{p}}{2 \tau_{c}} \\
& \times \log _{2}\left(1+\frac{E_{r} \sigma_{A R, i}^{4} \sigma_{R B, i}^{4}}{\sum_{n=1}^{N}\left(\sigma_{A R, n}^{2} \sigma_{R B, n}^{2}\left(\sigma_{A R, n}^{2}+\sigma_{R B, n}^{2}\right)\right)}\right) .
\end{aligned}
$$

Similar to the analysis in Corollary 2, if the down-scaling of the relay transmit power in the second phase is faster than that of the users' transmit power in the first phase, the limit of $R_{A, i}$ will only depend on the noise at the users.

\section{Scenario $C$}

In scenario $\mathrm{C}$, all the transmit powers scale with the number of relay antennas, i.e., $p_{u}=\frac{E_{u}}{M^{\alpha}}, p_{r}=\frac{E_{r}}{M^{\beta}}$, and $p_{p}=\frac{E_{p}}{M^{\gamma}}$, with $\alpha \geq 0, \beta \geq 0$, and $\gamma>0, E_{u}, E_{r}$, and $E_{p}$ are constants. This is the most general scenario where we are able to flexibly adjust the transmit powers of the considered system to maintain the desired performance.

Now define the following conditions, namely, Condition V: $\frac{M^{\alpha}}{E_{u} \beta_{R B, i}^{2}}+\frac{M^{\beta}}{E_{r} \beta_{A R, i}^{4} \beta_{R B, i}^{4}} \sum_{n=1}^{N} \beta_{A R, n}^{2} \beta_{R B, n}^{2}\left(\beta_{A R, n}^{2}+\beta_{R B, n}^{2}\right) \gg$ $\frac{1}{\beta_{R B, i}}+\frac{1}{\beta_{A R, i}}+\frac{4 \beta_{A R, i}}{\beta_{R B, i}^{2}}+\sum_{j \neq i}\left(\frac{\beta_{A R, j}}{\beta_{R B, i}^{2}}+\frac{\beta_{A R, j}^{4} \beta_{R B, j}^{2}}{\beta_{A R, i}^{3} \beta_{R B, i}^{4}}\right)+$

$\sum_{j \neq i}\left(\frac{\beta_{R B, j}}{\beta_{R B, i}^{2}}+\frac{\beta_{A R, j}^{2} \beta_{R B, j}^{4}}{\beta_{A R, i}^{3} \beta_{R B, i}^{4}}\right)$,
Condition VI:

$\frac{1}{E_{u}} \gg \frac{M^{\beta+\gamma-1}}{E_{r} \beta_{A R, i}^{4} \beta_{R B, i}^{2}} \sum_{n=1}^{N} \beta_{A R, n}^{2} \beta_{R B, n}^{2}\left(\beta_{A R, n}^{2}+\beta_{R B, n}^{2}\right)$, and Condition VII:

$\frac{M^{\alpha+\gamma-1}}{E_{u}} \ll \frac{1}{E_{r} \beta_{A R, i}^{4} \beta_{R B, i}^{2}} \sum_{n=1}^{N} \beta_{A R, n}^{2} \beta_{R B, n}^{2}\left(\beta_{A R, n}^{2}+\beta_{R B, n}^{2}\right)$, then we have:

Theorem 5: For fixed and finite $E_{u}, E_{r}$, and $E_{p}$, when $p_{u}=\frac{E_{u}}{M^{\alpha}}, p_{r}=\frac{E_{r}}{M^{\beta}}$, and $p_{p}=\frac{E_{p}}{M^{\gamma}}$, with $\alpha \geq 0, \beta \geq 0$, and $\gamma>0$, as $M \rightarrow \infty$ and Conditions I and V hold, we have (38), shown on the top of the next page.

Theorem 5 reveals the coupled relationship between the training power and user (or relay) transmit power. When $\alpha+\gamma>1$ and/or $\beta+\gamma>1, R_{A, i}$ converges to zero, due 


$$
R_{A, i}-\frac{\tau_{c}-\tau_{p}}{2 \tau_{c}} \log _{2}\left(1+\frac{1}{\frac{M^{\alpha+\gamma-1}}{\tau_{p} E_{p} E_{u} \beta_{R B, i}^{2}}+\frac{M^{\beta+\gamma-1}}{\tau_{p} E_{p} E_{r} \beta_{A R, i}^{4} \beta_{R B, i}^{4}} \sum_{n=1}^{N} \beta_{A R, n}^{2} \beta_{R B, n}^{2}\left(\beta_{A R, n}^{2}+\beta_{R B, n}^{2}\right)}\right) \stackrel{M \rightarrow \infty}{\longrightarrow} 0 .
$$

to either poor estimation accuracy or low user/relay transmit power. On the other hand, when $0<\alpha+\gamma<1$ and $0<\beta+\gamma<1, R_{A, i}$ grows without bound. Only if $\alpha+\gamma=1$ and/or $\beta+\gamma=1, R_{A, i}$ converges to a non-zero limit. In the following, we take a closer look at these particular cases of interest.

Corollary 4: For fixed and finite $E_{u}, E_{r}$, and $E_{p}$, when $\alpha=\beta>0$ and $\alpha+\gamma=1$, namely, $p_{u}=\frac{E_{u}}{M^{\alpha}}, p_{r}=\frac{E_{r}}{M^{\beta}}$, and $p_{p}=\frac{E_{p}}{M^{\gamma}}$, with $\gamma>0$, as $M \rightarrow \infty$ and Conditions I and V hold, the SE has the limit (39), shown on the top of the next page.

Corollary 4 suggests that no matter how $\alpha, \beta$, and $\gamma$ change, as long as the overall power reduction SE at the user/relay and pilot symbol remains the same, i.e., $\alpha+\gamma=1$, the same asymptotic SE can be attained. In other words, it is possible to balance between the pilot symbol power to the user/relay transmit power.

Corollary 5: For fixed and finite $E_{u}, E_{r}$, and $E_{p}$, when $\alpha>\beta \geq 0$ and $\alpha+\gamma=1$, namely, $p_{u}=\frac{E_{u}}{M^{\alpha}}, p_{r}=\frac{E_{r}}{M^{\beta}}$, and $p_{p}=\frac{E}{M_{p}}$, with $\gamma>0$, as $M \rightarrow \infty$ and Conditions I, V, and VI hold, the SE has the limit

$$
R_{A, i} \rightarrow \frac{\tau_{c}-\tau_{p}}{2 \tau_{c}} \log _{2}\left(1+\tau_{p} E_{p} E_{u} \beta_{R B, i}^{2}\right) .
$$

Corollary 5 shows the same trade-off between $\alpha$ and $\gamma$ as in Corollary 4. However, similar to Corollary 2, the SE is only related to the noise at the relay.

Corollary 6: For fixed and finite $E_{u}, E_{r}$, and $E_{p}$, when $0 \leq \alpha<\beta$ and $\beta+\gamma=1$, namely, $p_{u}=\frac{E_{u}}{M^{\alpha}}, p_{r}=\frac{E_{r}}{M^{\beta}}$, and $p_{p}=\frac{E_{p}}{M \gamma}$, with $\gamma>0$, as $M \rightarrow \infty$ and Conditions I, V, and VII hold, the SE has the limit

$$
\begin{aligned}
& R_{A, i} \rightarrow \frac{\tau_{c}-\tau_{p}}{2 \tau_{c}} \\
& \times \log _{2}\left(1+\frac{\tau_{p} E_{p} E_{r} \beta_{A R, i}^{4} \beta_{R B, i}^{4}}{\sum_{n=1}^{N} \beta_{A R, n}^{2} \beta_{R B, n}^{2}\left(\beta_{A R, n}^{2}+\beta_{R B, n}^{2}\right)}\right) .
\end{aligned}
$$

Corollary 6 indicates that when we cut down the transmit power of the relay $p_{r}$ more compared with the transmit power of each user $p_{u}$, i.e., $0 \leq \alpha<\beta$, to obtain a constant limit SE, the trade-off between $p_{r}$ and the transmit power of the relay each pilot symbol $p_{p}$ should be satisfied, namely, $\beta+\gamma=1$. This trade-off provides valuable insights, since we can adjust $p_{r}$ and $p_{p}$ flexibly based on different demands, to meet the same limit. In addition, Corollary 6 also shows that $R_{A, i}$ is an increasing function with respect to $E_{p}$ and $E_{r}$, while a decreasing function of $N$. In other words, when the number of user pairs $N$ increases, the relay and/or each pilot symbol should increase their power in order to maintain the same performance. This is due to the fact that a larger transmit power of the relay and/or more accurate channel estimation can compensate the individual SE loss caused by stronger inter-user interference.

\section{Power Allocation}

In this section, we formulate a power allocation problem maximizing the sum SE subject to a total power constraint, i.e., $\sum_{i=1}^{N}\left(p_{A, i}+p_{B, i}\right)+p_{r} \leq P$. For notational simplicity, we define $\mathcal{N} \triangleq\{1, \ldots, N\}, \mathbf{p}_{A} \triangleq\left[p_{A, 1}, \ldots, p_{A, N}\right]^{T}$, and $\mathbf{p}_{B} \triangleq$ $\left[p_{B, 1}, \ldots, p_{B, N}\right]^{T}$.

For mathematical tractability, instead of using the sum SE expression in Theorem 1, we work with the large-scale approximation from Theorem 2 which turns out to be tight for even moderate $M$ in the simulation results of Section VI-A. Thus, the power allocation optimization problem is formulated as [25]

$$
\begin{array}{ll}
\underset{\mathbf{p}_{A}, \mathbf{p}_{B}, p_{r}}{\operatorname{maximize}} & \sum_{i=1}^{N}\left(\tilde{R}_{A, i}+\tilde{R}_{B, i}\right) \\
\text { subject to } & \sum_{i=1}^{N}\left(p_{A, i}+p_{B, i}\right)+p_{r} \leq P, \\
& \mathbf{0} \leq \mathbf{p}_{A} \leq p_{0} \mathbf{1}, \mathbf{0} \leq \mathbf{p}_{B} \leq p_{0} \mathbf{1}, 0 \leq p_{r} \leq p_{1},
\end{array}
$$

where $\tilde{R}_{B, i}$ is the large-scale approximation for the SE of $\mathrm{T}_{B, i}$, which can be derived in a similar fashion, $p_{0}$ and $p_{1}$ are the peak power constraints of $p_{A, i}\left(p_{B, i}\right)$ and $p_{r}$, respectively.

Since $\log (\cdot)$ is an increasing function, (42) can be equivalently reformulated as:

$$
\begin{aligned}
\mathcal{P}_{1}: \underset{\substack{\mathbf{p}_{A}, \mathbf{p}_{B}, p_{r} \\
\gamma_{A}, \gamma_{B}}}{\operatorname{minimize}} & \prod_{i=1}^{N}\left(1+\gamma_{A, i}\right)^{-1}\left(1+\gamma_{B, i}\right)^{-1} \\
\text { subject to } \quad & \gamma_{A, i} \leq \frac{p_{B, i}}{\xi_{i}}, \gamma_{B, i} \leq \frac{p_{A, i}}{\tilde{\xi}_{i}}, i \in \mathcal{N} \\
& \sum_{i=1}^{N}\left(p_{A, i}+p_{B, i}\right)+p_{r} \leq P, \\
& \mathbf{0} \leq \mathbf{p}_{A} \leq p_{0} \mathbf{1}, \mathbf{0} \leq \mathbf{p}_{B} \leq p_{0} \mathbf{1}, 0 \leq p_{r} \leq p_{1},
\end{aligned}
$$

where $\gamma_{A} \triangleq\left[\gamma_{A, 1}, \ldots, \gamma_{A, N}\right]^{T}, \gamma_{B} \triangleq\left[\gamma_{B, 1}, \ldots, \gamma_{B, N}\right]^{T}$, $\gamma_{A R, i}$ and $\gamma_{R B, i}$ are considered as the signal-to-interferenceplus-noise ratio (SINR) of $\tilde{R}_{A, i}$ and $\tilde{R}_{B, i}$, respectively. Also, $\xi_{i}$ and $\tilde{\xi}_{i}$ are respectively given by

$$
\begin{aligned}
\xi_{i}=\sum_{j=1}^{N}\left(a_{i, j} p_{A, j}+b_{i, j} p_{B, j}\right) & \\
& +p_{r}^{-1} \sum_{j=1}^{N}\left(c_{i, j} p_{A, j}+d_{i, j} p_{B, j}\right)+e_{i},
\end{aligned}
$$




$$
R_{A, i} \rightarrow \frac{\tau_{c}-\tau_{p}}{2 \tau_{c}} \log _{2}\left(1+\frac{1}{\frac{1}{\tau_{p} E_{p} E_{u} \beta_{R B, i}^{2}}+\frac{1}{\tau_{p} E_{p} E_{r} \beta_{A R, i}^{4} \beta_{R B, i}^{4}} \sum_{n=1}^{N}\left(\beta_{A R, n}^{2} \beta_{R B, n}^{2}\left(\beta_{A R, n}^{2}+\beta_{R B, n}^{2}\right)\right)}\right)
$$

and

$$
\begin{aligned}
\tilde{\xi}_{i}=\sum_{j=1}^{N}\left(\tilde{a}_{i, j} p_{A, j}+\tilde{b}_{i, j} p_{B, j}\right) & \\
& +p_{r}^{-1} \sum_{j=1}^{N}\left(\tilde{c}_{i, j} p_{A, j}+\tilde{d}_{i, j} p_{B, j}\right)+\tilde{e}_{i}
\end{aligned}
$$

where

$$
\begin{aligned}
a_{i, j} & =\frac{1}{M} \begin{cases}\frac{4 \beta_{A R, i}}{\sigma_{R B, i}^{2}}, & j=i, \\
\frac{\beta_{A R, j}}{\sigma_{R B, i}^{2}}+\frac{\sigma_{A R, j}^{4} \sigma_{R B, j}^{2} \beta_{A R, i}}{\sigma_{A R, i}^{4} \sigma_{R B, i}^{4}}, & j \neq i,\end{cases} \\
b_{i, j} & =\frac{1}{M} \begin{cases}\frac{\beta_{R B, i}}{\sigma_{R B, i}^{2}}+\frac{\beta_{A R, i}}{\sigma_{A R, i}^{2},}, & j=i, \\
\frac{\beta_{R B, j}}{\sigma_{R B, i}^{2}}+\frac{\sigma_{A R, j}^{2} \sigma_{R B, j}^{4} \beta_{A R, i}}{\sigma_{A R, i}^{4} \sigma_{R B, i}^{4}}, & j \neq i,\end{cases} \\
c_{i, j} & =\frac{\sigma_{A R, j}^{4} \sigma_{R B, j}^{2}}{M \sigma_{A R, i}^{4} \sigma_{R B, i}^{4}}, \\
d_{i, j} & =\frac{\sigma_{A R, j}^{2} \sigma_{R B, j}^{4}}{M \sigma_{A R, i}^{4} \sigma_{R B, i}^{4}}, \\
e_{i} & =\frac{1}{M \sigma_{R B, i}^{2}},
\end{aligned}
$$

and $\tilde{a}_{i, j}, \tilde{b}_{i, j}, \tilde{c}_{i, j}, \tilde{d}_{i, j}, \tilde{e}_{i}$ are obtained by replacing the subscripts "AR", "RB" with "RB", "AR" in $b_{i, j}, a_{i, j}, d_{i, j}, c_{i, j}$, $e_{i}$, respectively. Note that we have replaced the equality "=" with " $\leq$ " in the first two constraints of problem $\mathcal{P}_{1}$; however, this does not change or relax the original problem (42), since the objective function is decreasing with $\gamma_{A R, i}$ and $\gamma_{R B, i}$. Therefore, we can guarantee that these two constraints must be active at any optimal solution of $\mathcal{P}_{1}$.

The above problem $\mathcal{P}_{1}$ is identified as a complementary geometric programming problem, which is nonconvex [30], hence, the optimal solution is intractable. Responding to this, we propose an efficient suboptimal solution for problem $\mathcal{P}_{1}$, which significantly outperforms the uniform power allocation scheme. Specifically, noticing that if the objective function is a monomial function, the problem $\mathcal{P}_{1}$ becomes a standard GP problem, and can be solved efficiently with standard optimization tools such as CVX [31] or ggplab [32]. The key idea is to use a monomial function $\omega_{X, i}\left(\gamma_{X, i}\right)^{\mu_{X, i}}$ to approximate $1+\gamma_{X, i}$ near an arbitrary point $\hat{\gamma}_{X, i}>0$, where $X \in\{A, B\}$, $\mu_{X, i}=\frac{\hat{\gamma}_{X, i}}{1+\hat{\gamma}_{X, i}}$ and $\omega_{X, i}=\left(\hat{\gamma}_{X, i}\right)^{-\mu_{X, i}}\left(1+\hat{\gamma}_{X, i}\right)$. At each iteration, the GP is obtained by replacing the posynomial objective function with its best local monomial approximation near the solution obtained at the previous iteration. Then, a local optimum of the original problem $\mathcal{P}_{1}$ can be found by solving a sequence of GPs, capitalizing on the technique proposed in [33, Lemma 1]. ${ }^{3}$ Now, we outline the successive approximation algorithm to solve the original problem $\mathcal{P}_{1}$ in the following.

Algorithm 1 Successive approximation algorithm for $\mathcal{P}_{1}$

1) Initialization. Define a tolerance $\epsilon$ and parameter $\theta$. Set $k=1$, the initial values of $\hat{\gamma}_{A, i}$ and $\hat{\gamma}_{B, i}$ are chosen according to the SINR in Theorem 2 when letting $p_{A, i}=p_{B, i}=\frac{P}{4 N}$, and $p_{r}=\frac{P}{2}$.

2) Iteration $k$. Compute $\mu_{A, i}=\frac{\hat{\gamma}_{A, i}}{1+\hat{\gamma}_{A, i}}$ and $\mu_{B, i}=\frac{\hat{\gamma}_{B, i}}{1+\hat{\gamma}_{B, i}}$. Then, solve the following GP problem:

$$
\begin{aligned}
\mathcal{P}_{2}: \underset{\substack{\mathbf{p}_{A}, \mathbf{p}_{B}, p_{r} \\
\gamma_{A}, \gamma_{B}}}{\operatorname{sinimize}} & \prod_{i=1}^{N}\left(\gamma_{A, i}\right)^{-\mu_{A, i}\left(\gamma_{B, i}\right)^{-\mu_{B, i}}} \\
\text { subject to } & \theta^{-1} \hat{\gamma}_{A, i} \leq \gamma_{A, i} \leq \theta \hat{\gamma}_{A, i}, i \in \mathcal{N} \\
& \theta^{-1} \hat{\gamma}_{B, i} \leq \gamma_{B, i} \leq \theta \hat{\gamma}_{B, i}, i \in \mathcal{N} \\
& \gamma_{A, i} p_{B, i}^{-1} \xi_{i} \leq 1, i \in \mathcal{N} \\
& \gamma_{B, i} p_{A, i}^{-1} \tilde{\xi}_{i} \leq 1, i \in \mathcal{N} \\
& \sum_{i=1}^{N}\left(p_{A, i}+p_{B, i}\right)+p_{r} \leq P, \\
& \mathbf{0} \leq \mathbf{p}_{A} \leq p_{0} \mathbf{1}, \mathbf{0} \leq \mathbf{p}_{B} \leq p_{0} \mathbf{1}, 0 \leq p_{r} \leq p_{1} .
\end{aligned}
$$

Denote the optimal solutions by $\gamma_{A, i}^{(k)}$ and $\gamma_{B, i}^{(k)}, i \in \mathcal{N}$.

3) Stopping criterion. If $\max _{i}\left|\gamma_{A, i}^{(k)}-\hat{\gamma}_{A, i}\right|<\epsilon$ and/or $\max _{i}\left|\gamma_{B, i}^{(k)}-\hat{\gamma}_{B, i}\right|<\epsilon$, stop; otherwise, go to step 4).

4) Update initial values. Set $\hat{\gamma}_{A, i}=\gamma_{A, i}^{(k)}$ and $\hat{\gamma}_{B, i}=\gamma_{B, i}^{(k)}$, and $k=k+1$. Go to step 2).

Note that we have neglected $\omega_{A, i}$ and $\omega_{B, i}$ in the objective function of $\mathcal{P}_{2}$, since they are constants and do not affect the problem solution at each iteration. Also, some trust region constraints, i.e., the first two constraints, are added, which limit how much the variables are allowed to differ from the current guess $\hat{\gamma}_{A, i}$ and $\hat{\gamma}_{B, i}$. The limit of any convergent sequence generated by Algorithm 1 is a Karush-Kuhn-Tucker point, and the detailed proof can be found in [35]. The parameter $\theta>1$ controls the desired accuracy. More precisely, when $\theta$ is close to 1 it provides good accuracy for the monomial approximation but with slower convergence speed, and vice versa if $\theta$ is large. As discussed in [33], [36], [37], $\theta=1.1$ offers a good tradeoff between the accuracy and convergence speed.

Regarding the complexity of algorithm 1, we notice that algorithm 1 is executed by solving a sequence of GP problems. According to [34], GP can be solved by the interior point

\footnotetext{
${ }^{3}$ During the submission of the current work, an independent work has appeared in [25], which also uses the technique proposed in [33] to tackle the power allocation issue in the context of multipair massive MIMO two-way relaying systems.
} 
method with provably polynomial time complexity. Also, it can be efficiently implemented with high-quality software such as the MOSEK package.

To gain further insights, we now consider the special case when all the users transmit with the same power, i.e., $p_{A, i}=$ $p_{B, i}=p_{u}$. Also, we remove the peak power constraints by assuming that $p_{0}$ and $p_{1}$ are very high. Then, the optimization problem (42) reduces to

$$
\begin{aligned}
\mathcal{P}_{3}: \underset{p_{u}, p_{r}}{\operatorname{maximize}} & \sum_{i=1}^{N}\left(\tilde{R}_{A, i}+\tilde{R}_{B, i}\right) \\
\text { subject to } & 2 N p_{u}+p_{r} \leq P, \\
& p_{u} \geq 0, p_{r} \geq 0 .
\end{aligned}
$$

Theorem 6: $\mathcal{P}_{3}$ is a convex optimization problem.

Proof: See Appendix B.

Since the optimization problem $\mathcal{P}_{3}$ is convex, the optimal solutions $p_{u}^{\mathrm{opt}} \in\left(0, \frac{P}{2 N}\right]$ and $p_{r}^{\mathrm{opt}} \in(0, P]$ maximizing the sum SE can be obtained efficiently by adopting some standard techniques, such as the bisection method with respect to $P$. However, we cannot directly obtain closed-form expressions of $p_{u}^{\mathrm{opt}}$ and $p_{r}^{\mathrm{opt}}$, since the objective function relies on the statistical characteristics of all the channel vectors. In order to simplify the analysis and provide some further insights, we assume that all the users have the same large-scale fading, e.g., $\beta_{A R, i}=\beta_{R B, i}=1$, thereby resulting in $\sigma_{A, i}^{2}=\sigma_{B, i}^{2}=\sigma^{2}$, $\tilde{\sigma}_{A, i}^{2}=\tilde{\sigma}_{B, i}^{2}=\tilde{\sigma}^{2}, \tilde{R}_{A, i}=\tilde{R}_{B, i}$, and then the optimization problem $\mathcal{P}_{3}$ can be analytically solved in the following theorem:

Theorem 7: The optimization problem $\mathcal{P}_{3}$ for the scenario where all the users have the same large-scale fading, e.g., $\beta_{A R, i}=\beta_{R B, i}=1$ is solved by

$$
\left\{\begin{array}{l}
p_{u}^{\mathrm{opt}}=\frac{P}{4 N}, \\
p_{r}^{\mathrm{opt}}=\frac{P}{2} .
\end{array}\right.
$$

Theorem 7 suggests that, for a given power budget $2 N p_{u}+$ $p_{r} \leq P$, half of the total power should be allocated to the relay regardless of the number of users, and the remaining half should be equally allocated to the $2 N$ users. Such a symmetric power allocation strategy is rather intuitive due to the symmetric system setup. In addition, it can be directly inferred that the optimal power $p_{u}^{\text {opt }}$ decreases monotonically by increasing the number of user pairs $N$, which serves as a useful guideline for practical system design.

\section{Numerical Results}

In this section, we present numerical results to validate the previous analytical results. For all illustrative examples, the following set of parameters are used in simulation. Unless otherwise specified, the length of the coherence interval is $\tau_{c}=196$ symbols, chosen by the LTE standard. The length of the pilot sequences is $\tau_{p}=2 N$ which is the minimum requirement. For simplicity, we set the large-scale fading coefficient $\beta_{A R}=\beta_{R B}=1$, and assume that each user has the same transmit power, i.e., $p_{A, i}=p_{B, i}=p_{u}$.

\section{A. Validation of analytical expressions}

Fig. 2 shows the sum SE versus the transmit power of each user $p_{u}$ for different number of relay antennas with $p_{p}=p_{u}$ and $p_{r}=2 N p_{u}$. Note that the "Approximations" curves are obtained by using (23), and the "Numerical results" curves are generated according to (16) by averaging over $10^{4}$ independent channel realizations, respectively. As can be readily observed, the large-scale approximations are very accurate, especially for large antenna arrays. Also, as expected, increasing the number of relay antennas significantly yields higher SE.

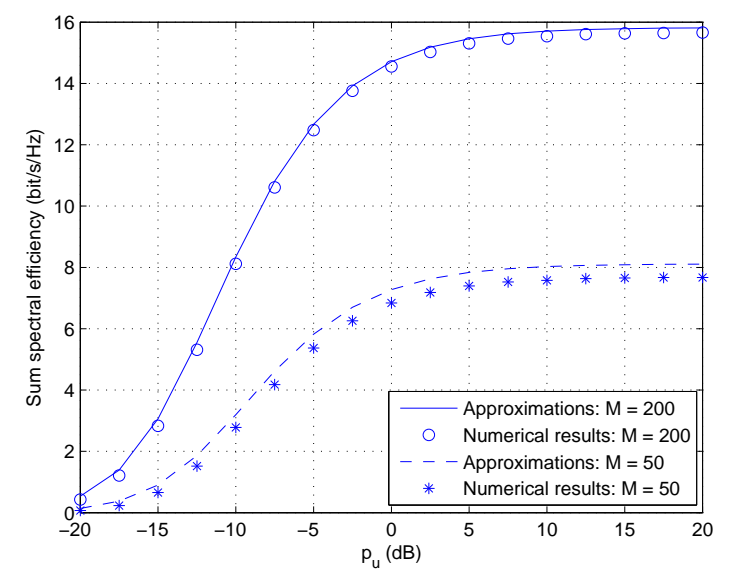

Fig. 2: Sum SE versus $p_{u}$ for $N=5, p_{p}=p_{u}$ and

$$
p_{r}=2 N p_{u} \text {. }
$$

\section{B. Power scaling}

In this subsection, we present numerical simulation results to verify the previous power scaling law analysis for three different scenarios, and demonstrate the power efficiency of using large number of antennas at the relay.

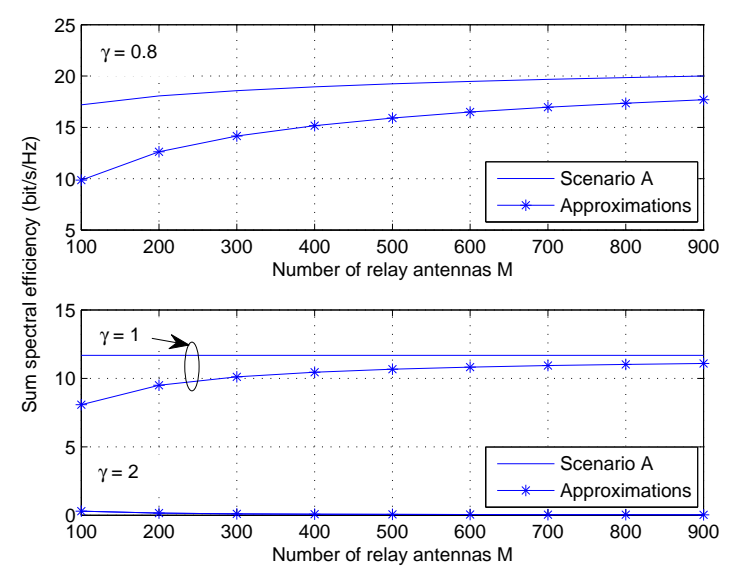

Fig. 3: Sum SE versus the number of relay antennas $M$ for $N=5, p_{u}=10 \mathrm{~dB}, p_{r}=20 \mathrm{~dB}$, and $p_{p}=E_{p} / M^{\gamma}$ with $E_{p}=10 \mathrm{~dB}$. 
1) Scenario A: Fig. 3 verifies the analytical results for Scenario A. Note that the curves labelled as "Scenario A" are plotted according to Theorems 3. We can see that when $M$ is large, the two curves are almost overlapped, which means that the previous asymptotic analysis is very accurate. In addition, when $\gamma>1$, e.g., $\gamma=2$, the SE gradually approaches zero. In contrast, when $0<\gamma<1$, e.g., $\gamma=0.8$, the $\mathrm{SE}$ grows unbounded. Finally, when $\gamma=1$, the SE converges to a nonzero limit.

2) Scenario B: Fig. 4 investigates how the transmit power of each user $p_{u}=\frac{E_{u}}{M^{\alpha}}$ and the transmit power of the relay $p_{r}=\frac{E_{r}}{M^{\beta}}$ scale with $M$. To fully evaluate the SE behavior, we consider three different cases based on the values of $\alpha$ and $\beta$, namely, 1) Case I: $\alpha=\beta=1$; 2) Case II: $\alpha=1, \beta=0.2$; 3) Case III: $\alpha=0.4, \beta=1$. Note that the curves labelled as "Scenario B" are generated by using Theorem 4, while the curves labelled as "Scenario B-Case X" with $X \in\{$ I, II, III $\}$ are plotted according to Corollaries 1-3, respectively. Fig. 4(a) shows that the sum SE saturates in the asymptotical large $M$ regime for all the three cases, which agrees with Corollaries 13. Furthermore, Case I has the lowest SE due to simultaneously cutting the transmit powers of each user and of the relay, while Case II and Case III achieve the same performance due to the setting of $E_{r}=2 N E_{u}$.

Fig. 4(b) illustrates the system performance when the transmit power down-scaling is either too aggressive or too moderate. As expected, as the number of relay antennas increases, the sum SE gradually reduces to zero for $\alpha>1, \beta \geq 0$, $\alpha \geq 0, \beta>1$, or $\alpha>1, \beta>1$. However, the speed of reduction varies significantly depending on the scaling parameters. The larger the scaling parameters, the faster the decay of the SE. In contrast, if we cut down the transmit powers of each user and of the relay moderately, the sum SE grows unboundedly.

3) Scenario $C$ : Fig. 5 presents the tradeoff between the user/relay power and the pilot symbol power. We set two examples, i.e., $\alpha=1.3, \beta=1.1, \gamma=0.5$ and $\alpha=0.8$, $\beta=0.6, \gamma=1$, which satisfy $\alpha+\gamma=1.8$ and $\beta+\gamma=1.6$. As predicted, the sum SE converges to zero for too aggressive power down-scaling. Moreover, the gaps between the two sets of curves narrow down with $M$ and eventually vanish. This indicates that as long as $\alpha+\gamma$ and $\beta+\gamma$ are the same, the asymptotic sum SE remains unchanged. Now, let us focus on the two curves associated with $N=5$. Interestingly, we see that the curve associated with $\gamma=0.5$ yields better performance in the finite antenna regime, despite the fact that the user or relay power is over-reduced compared to the $\gamma=1$ case, which suggests that the channel estimation accuracy is crucially important for the system. The same behavior appears for the unbounded SE scenario where $\alpha+\gamma=0.9$ and $\beta+\gamma=0.8$.

\section{Power allocation}

Fig. 6 illustrates the impact of the optimal power allocation scheme on the sum SE. The different large-scale fading parameters are arbitrarily generated by $\beta_{A R, i}=z_{i}\left(r_{A R, i} / r_{0}\right)^{\alpha}$ and $\beta_{R B, i}=z_{i}\left(r_{B R, i} / r_{0}\right)^{\alpha}$, where $z_{i}$ is a log-normal random

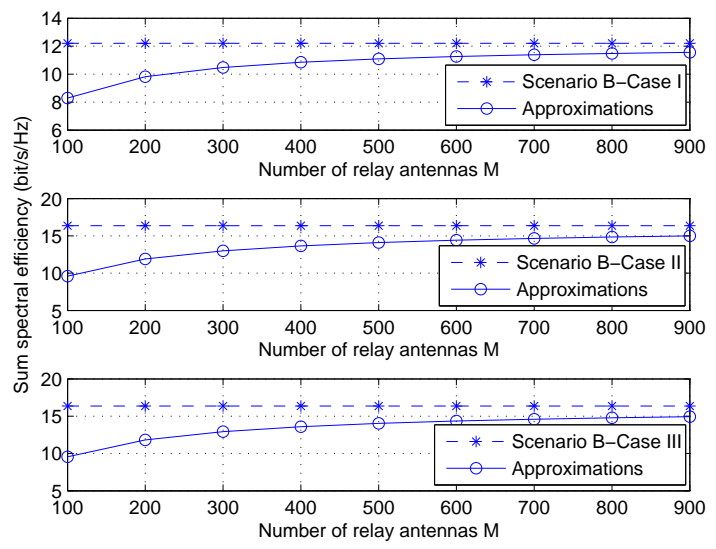

(a) Case I II, and III

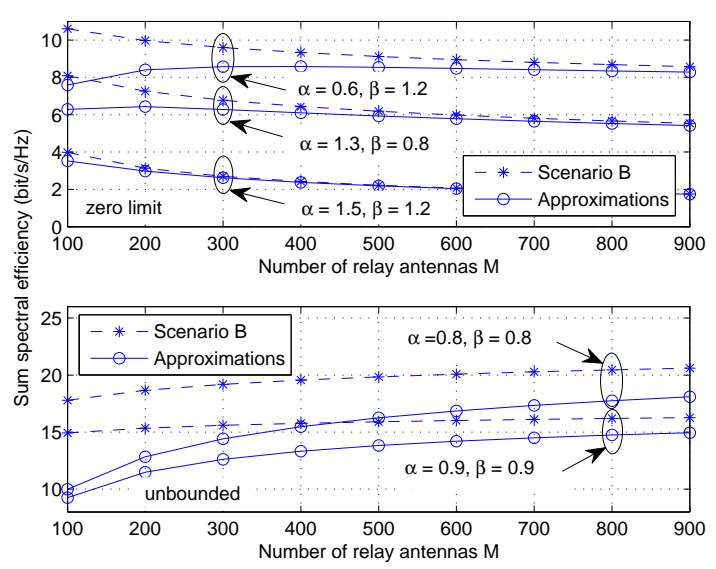

(b) Zero limit and unbounded

Fig. 4: Sum SE versus the number of relay antennas $M$ for $p_{p}=10 \mathrm{~dB}, p_{u}=E_{u} / M^{\alpha}$ with $E_{u}=10 \mathrm{~dB}$, and $p_{r}=E_{r} / M^{\beta}$ with $E_{r}=20 \mathrm{~dB}$.

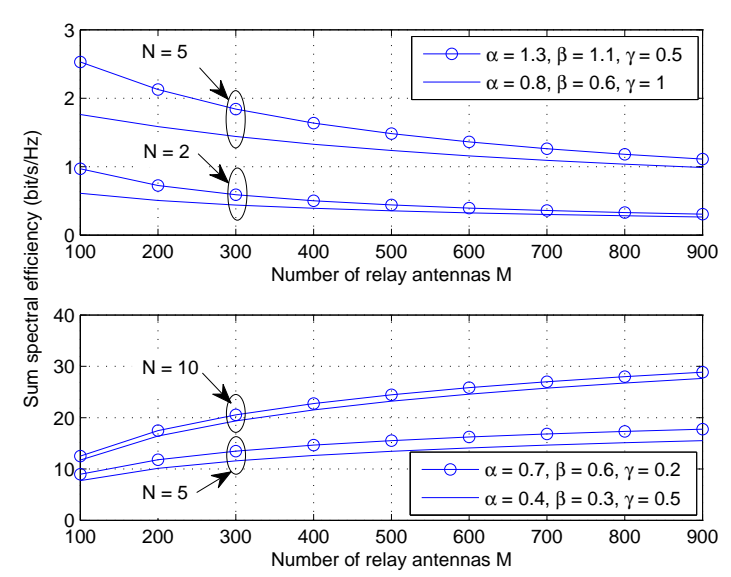

Fig. 5: Sum SE versus the number of relay antennas $M$ for $p_{u}=E_{u} / M^{\alpha}$ with $E_{u}=10 \mathrm{~dB}, p_{r}=E_{r} / M^{\beta}$ with $E_{r}=15 \mathrm{~dB}$, and $p_{p}=E_{p} / M^{\gamma}$ with $E_{p}=0 \mathrm{~dB}$.

variable with standard deviation $8 \mathrm{~dB}, r_{A R, i}$ and $r_{R B, i}$ are 
the locations of $\mathrm{T}_{A R, i}$ and $\mathrm{T}_{R B, i}$ from the relay, $\alpha=3.8$ is the path loss exponent, and $r_{0}$ denotes the guard interval which specifies the nearest distance between the users and the relay. The relay is located at the center of a cell with a radius of 1000 meters and $r_{0}=100$ meters. We choose $P=10 \mathrm{~dB}, p_{p}=10 \mathrm{~dB}, N=5, p_{0}=P / 2 N, p_{1}=P$, $\beta_{A R}=[0.2688,0.0368,0.00025,0.1398,0.0047]$, and $\beta_{R B}=$ $[0.0003,0.00025,0.0050,0.0794,0.0001]$. The optimal power allocation curves are generated by Algorithm 1. Also, we plot the sum SE with uniform power allocation as a benchmark scheme for comparison. As can be observed, the optimal power allocation policy provides $23.1 \%$ SE enhancement when $M=300$. Moreover, by focusing on the case where every user has the same transmit power, i.e, $p_{A, i}=p_{B, i}=p_{u}$, we can see that the optimal user transmit power is a decreasing function with respect to the number of user pairs $N$, which aligns with Theorem 7.

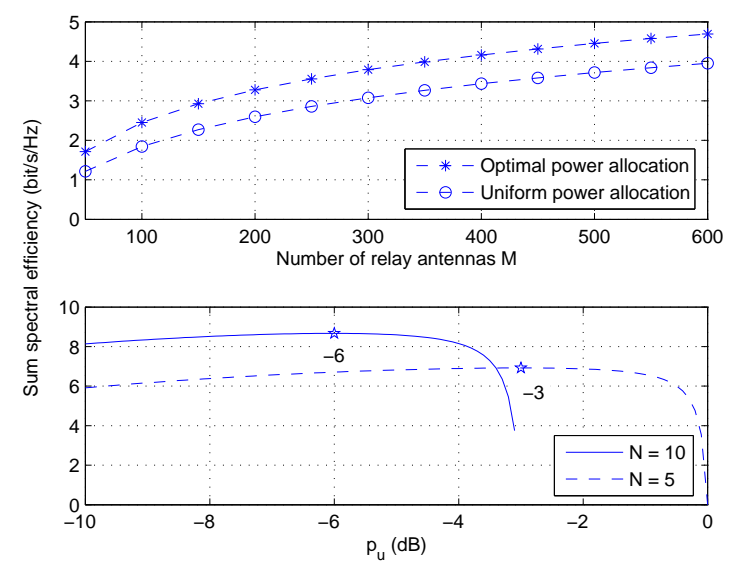

Fig. 6: Sum SE for a given power budget $P=10 \mathrm{~dB}$.

\section{CONCLUSION}

We have investigated the sum SE of a multipair two-way half-duplex relaying system employing the MR scheme taking into account of realistic CSI. In particular, a closed-form expression and a large-scale approximation were derived for the sum SE. Moreover, different power scaling laws of the system were characterized, which showed that the transmit powers of user, relay, and pilot symbol can be scaled down inversely proportional to the number of relay antennas. In addition, it was revealed that there exists a fundamental tradeoff between the user/relay transmit power and pilot symbol power, which provides great flexibility for the design of practical systems. Finally, the transmit powers of each user and the relay were optimized to enhance the sum SE.

\section{APPENDIX A}

\section{PROOF OF THEOREM 1}

The end-to-end SINR given in (11) consists of five terms: 1) desired signal power $A_{i} ; 2$ ) estimation error $B_{i} ; 3$ ) residual self-interference $C_{i}$;4) inter-user interference $\left.D_{i} ; 5\right)$ compound noise $E_{i}$.
1) Compute $A_{i}$ :

$$
\begin{aligned}
& \mathrm{E}\left\{\mathbf{g}_{A R, i}^{T} \mathbf{F g}_{R B, i}\right\} \\
& =\mathrm{E}\left\{\left|\hat{\mathbf{g}}_{A R, i}^{H} \hat{\mathbf{g}}_{R B, i}\right|^{2}+\left\|\hat{\mathbf{g}}_{A R, i}\right\|^{2}\left\|\hat{\mathbf{g}}_{R B, i}\right\|^{2}\right\} \\
& =M(M+1) \sigma_{A R, i}^{2} \sigma_{R B, i}^{2} .
\end{aligned}
$$

Consequently, we obtain

$$
A_{i}=p_{B, i} M^{2}(M+1)^{2} \sigma_{A R, i}^{4} \sigma_{R B, i}^{4} .
$$

2) Compute $B_{i}$ :

$$
\begin{aligned}
& \mathrm{E}\left\{\left|\mathbf{g}_{A R, i}^{T} \mathbf{F} \mathbf{g}_{R B, i}\right|^{2}\right\} \\
& =\mathrm{E}\left\{\sum_{n=1}^{N} \sum_{l=1}^{N} \mathbf{g}_{A R, i}^{T} \mathbf{C}_{n} \mathbf{g}_{R B, i} \mathbf{g}_{R B, i}^{H} \mathbf{C}_{l}^{H} \mathbf{g}_{A R, i}^{*}\right\},
\end{aligned}
$$

where $\mathbf{C}_{n}=\left(\hat{\mathbf{g}}_{R B, n}^{*} \hat{\mathbf{g}}_{A R, n}^{H}+\hat{\mathbf{g}}_{A R, n}^{*} \hat{\mathbf{g}}_{R B, n}^{H}\right)$, which can be decomposed into three different cases:

a) for $n \neq l \neq i$, we have $\mathrm{E}\left\{\left|\mathbf{g}_{A R, i}^{T} \mathbf{F} \mathbf{g}_{R B, i}\right|^{2}\right\}=0$.

b) for $n=l \neq i$, we have

$$
\begin{aligned}
& \mathrm{E}\left\{\left|\mathbf{g}_{A R, i}^{T} \mathbf{F g}_{R B, i}\right|^{2}\right\} \\
& =2 M(M+1) \beta_{A R, i} \beta_{R B, i} \sum_{n \neq i} \sigma_{A R, n}^{2} \sigma_{R B, n}^{2} .
\end{aligned}
$$

c) for $n=l=i$, we have

$$
\begin{aligned}
& \mathrm{E}\left\{\left|\mathbf{g}_{A R, i}^{T} \mathbf{F} \mathbf{g}_{R B, i}\right|^{2}\right\} \\
& =\mathrm{E}\left\{\mathbf{g}_{A R, i}^{T} \hat{\mathbf{g}}_{R B, i}^{*} \hat{\mathbf{g}}_{A R, i}^{H} \mathbf{g}_{R B, i} \mathbf{g}_{R B, i}^{H} \hat{\mathbf{g}}_{A R, i} \hat{\mathbf{g}}_{R B, i}^{T} \mathbf{g}_{A R, i}^{*}\right\} \\
& +\mathrm{E}\left\{\mathbf{g}_{A R, i}^{T} \hat{\mathbf{g}}_{R B, i}^{*} \hat{\mathbf{g}}_{A R, i}^{H} \mathbf{g}_{R B, i} \mathbf{g}_{R B, i}^{H} \hat{\mathbf{g}}_{R B, i} \hat{\mathbf{g}}_{A R, i}^{T} \mathbf{g}_{A R, i}^{*}\right\} \\
& +\mathrm{E}\left\{\mathbf{g}_{A R, i}^{T} \hat{\mathbf{g}}_{A R, i}^{*} \hat{\mathbf{g}}_{R B, i}^{H} \mathbf{g}_{R B, i} \mathbf{g}_{R B, i}^{H} \hat{\mathbf{g}}_{A R, i} \hat{\mathbf{g}}_{R B, i}^{T} \mathbf{g}_{A R, i}^{*}\right\} \\
& +\mathrm{E}\left\{\mathbf{g}_{A R, i}^{T} \hat{\mathbf{g}}_{A R, i}^{*} \hat{\mathbf{g}}_{R B, i}^{H} \mathbf{g}_{R B, i} \mathbf{g}_{R B, i}^{H} \hat{\mathbf{g}}_{R B, i} \hat{\mathbf{g}}_{A R, i}^{T} \mathbf{g}_{A R, i}^{*}\right\} .
\end{aligned}
$$

The first term in (55) becomes

$$
\begin{aligned}
& \mathrm{E}\left\{\mathbf{g}_{A R, i}^{T} \hat{\mathbf{g}}_{R B, i}^{*} \hat{\mathbf{g}}_{A R, i}^{H} \mathbf{g}_{R B, i} \mathbf{g}_{R B, i}^{H} \hat{\mathbf{g}}_{A R, i} \hat{\mathbf{g}}_{R B, i}^{T} \mathbf{g}_{A R, i}^{*}\right\} \\
& =\mathrm{E}\left\{\left|\tilde{g}_{A R, i}\right|^{4}|| \hat{\mathbf{g}}_{R B, i}||^{4}\right\}+\tilde{\sigma}_{A R, i}^{2} \mathrm{E}\left\{\left|\tilde{g}_{A R, i}\right|^{2} \|\left.\hat{\mathbf{g}}_{R B, i}\right|^{4}\right\} \\
& +\tilde{\sigma}_{R B, i}^{2} \mathrm{E}\left\{\left|\tilde{g}_{R B, i}\right|^{2} \|\left.\hat{\mathbf{g}}_{A R, i}\right|^{4}\right\} \\
& +\tilde{\sigma}_{R B, i}^{2} \mathrm{E}\left\{\left|\hat{\mathbf{g}}_{R B, i}^{H} \mathbf{e}_{A R, i}\right|^{2}\right\} \mathrm{E}\left\{\|\left.\hat{\mathbf{g}}_{A R, i}\right|^{2}\right\},
\end{aligned}
$$

where $\tilde{g}_{A R, i} \triangleq \frac{\hat{\mathbf{g}}_{A R, i}^{H} \hat{\mathbf{g}}_{R B, i}}{\left\|\hat{\mathbf{g}}_{R B, i}\right\|}$ and $\tilde{g}_{R B, i} \triangleq \frac{\hat{\mathbf{g}}_{A R, i}^{H} \hat{\mathbf{g}}_{R B, i}}{\left\|\hat{\mathbf{g}}_{A R, i}\right\|}$. The vector $\tilde{g}_{A R, i}$ is a Gaussian random variable with zero mean and variance $\sigma_{A R, i}^{2}$ which is independent of $\hat{\mathbf{g}}_{R B, i}$, whereas $\tilde{g}_{R B, i}$ is a Gaussian random variable with zero mean and variance $\sigma_{R B, i}^{2}$ which is independent of $\hat{\mathbf{g}}_{A R, i}$. Therefore, (56) can be calculated as

$$
\begin{aligned}
& \mathrm{E}\left\{\mathbf{g}_{A R, i}^{T} \hat{\mathbf{g}}_{R B, i}^{*} \hat{\mathbf{g}}_{A R, i}^{H} \mathbf{g}_{R B, i} \mathbf{g}_{R B, i}^{H} \hat{\mathbf{g}}_{A R, i} \hat{\mathbf{g}}_{R B, i}^{T} \mathbf{g}_{A R, i}^{*}\right\} \\
& =M(M+1) \sigma_{A R, i}^{2} \sigma_{R B, i}^{2}\left(\beta_{A R, i} \sigma_{R B, i}^{2}+\beta_{R B, i} \sigma_{A R, i}^{2}\right) \\
& +M^{2} \sigma_{A R, i}^{2} \sigma_{R B, i}^{2} \tilde{\sigma}_{A R, i}^{2} \tilde{\sigma}_{R B, i}^{2} .
\end{aligned}
$$

Following the same procedure, the last three terms in (55) can also be derived. Finally, combining a), b), and c), we obtain $B_{i}$.

3) Compute $C_{i}$ : By utilizing the same technique as in the derivation of $B_{i}$, we obtain $C_{i}$. 
4) Compute $D_{i}$ :

$$
\begin{aligned}
& \mathrm{E}\left\{\left|\mathbf{g}_{A R, i}^{T} \mathbf{F} \mathbf{g}_{A R, j}\right|^{2}\right\} \\
& =\mathrm{E}\left\{\sum_{n=1}^{N} \sum_{l=1}^{N} \mathbf{g}_{A R, i}^{T} \mathbf{C}_{n} \mathbf{g}_{A R, j} \mathbf{g}_{A R, j}^{H} \mathbf{C}_{l}^{H} \mathbf{g}_{A R, i}^{*}\right\},
\end{aligned}
$$

which can be decomposed into six different cases: 0 .

a) for $n \neq l \neq i, j(j \neq i)$, we have $\mathrm{E}\left\{\left|\mathbf{g}_{A R, i}^{T} \mathbf{F} \mathbf{g}_{A R, j}\right|^{2}\right\}=$

b) for $n=l \neq i, j(j \neq i)$, we have

$$
\begin{aligned}
& \mathrm{E}\left\{\left|\mathbf{g}_{A R, i}^{T} \mathbf{F g}_{A R, j}\right|^{2}\right\} \\
& =2 M(M+1) \beta_{A R, i} \beta_{A R, j} \sum_{n \neq i, j} \sigma_{A R, n}^{2} \sigma_{R B, n}^{2} .
\end{aligned}
$$

c) for $n=l=i(j \neq i)$, we have

$$
\begin{aligned}
& \mathrm{E}\left\{\left|\mathbf{g}_{A R, i}^{T} \mathbf{F} \mathbf{g}_{A R, j}\right|^{2}\right\} \\
& =M \beta_{A R, j} \sigma_{A R, i}^{2} \sigma_{R B, i}^{2}(M+1)(M+3) \sigma_{A R, i}^{2} \\
& +M \beta_{A R, j} \sigma_{A R, i}^{2} \sigma_{R B, i}^{2} 2(M+1) \tilde{\sigma}_{A R, i}^{2} .
\end{aligned}
$$

d) for $n=l=j(j \neq i)$, we have

$$
\begin{aligned}
& \mathrm{E}\left\{\left|\mathbf{g}_{A R, i}^{T} \mathbf{F} \mathbf{g}_{A R, j}\right|^{2}\right\} \\
& =M \beta_{A R, i} \sigma_{A R, j}^{2} \sigma_{R B, j}^{2}(M+1)(M+3) \sigma_{A R, j}^{2} \\
& +M \beta_{A R, i} \sigma_{A R, j}^{2} \sigma_{R B, j}^{2} 2(M+1) \tilde{\sigma}_{A R, j}^{2} .
\end{aligned}
$$

e) for $n=i$ and $l=j(j \neq i)$, we have $\mathrm{E}\left\{\left|\mathbf{g}_{A R, i}^{T} \mathbf{F} \mathbf{g}_{A R, j}\right|^{2}\right\}=0$.

f) for $n=j$ and $l=i(j \neq i)$, we have $\mathrm{E}\left\{\left|\mathbf{g}_{A R, i}^{T} \mathbf{F} \mathbf{g}_{A R, j}\right|^{2}\right\}=0$.

Altogether, $\mathrm{E}\left\{\left|\mathbf{g}_{A R, i}^{T} \mathbf{F} \mathbf{g}_{A R, j}\right|^{2}\right\}$ is given by

$$
\begin{aligned}
& \mathrm{E}\left\{\mathbf{g}_{A R, i}^{T} \mathbf{F} \mathbf{g}_{A R, j}\right\} \\
& =2 M(M+1) \beta_{A R, i} \beta_{A R, j} \sum_{n \neq i, j} \sigma_{A R, n}^{2} \sigma_{R B, n}^{2} \\
& +M \beta_{A R, j} \sigma_{A R, i}^{2} \sigma_{R B, i}^{2}(M+1)(M+3) \sigma_{A R, i}^{2} \\
& +M \beta_{A R, j} \sigma_{A R, i}^{2} \sigma_{R B, i}^{2} 2(M+1) \tilde{\sigma}_{A R, i}^{2} \\
& +M \beta_{A R, i} \sigma_{A R, j}^{2} \sigma_{R B, j}^{2}(M+1)(M+3) \sigma_{A R, j}^{2} \\
& +M \beta_{A R, i} \sigma_{A R, j}^{2} \sigma_{R B, j}^{2} 2(M+1) \tilde{\sigma}_{A R, j}^{2} .
\end{aligned}
$$

Following the same technique as in deriving (62), we can obtain $\mathrm{E}\left\{\left|\mathbf{g}_{A R, i}^{T} \mathbf{F} \mathbf{g}_{R B, j}\right|^{2}\right\}$ and finally derive $D_{i}$.

5) Compute $E_{i}$ :

(a) Compute $\mathrm{E}\left\{\left\|\mathbf{g}_{A R, i}^{\mathrm{T}} \mathbf{F}\right\|^{2}\right\}$ :

Again, using the same technique as in the derivation of (62), we obtain

$$
\begin{aligned}
& \mathrm{E}\left\{\left\|\mathbf{g}_{A R, i}^{\mathrm{T}} \mathbf{F}\right\|^{2}\right\} \\
& =2 M(M+1) \beta_{A R, i} \sum_{n \neq i} \sigma_{A R, n}^{2} \sigma_{R B, n}^{2} \\
& +M \sigma_{A R, i}^{2} \sigma_{R B, i}^{2}(M+1)(M+3) \sigma_{A R, i}^{2} \\
& +M \sigma_{A R, i}^{2} \sigma_{R B, i}^{2} 2(M+1) \tilde{\sigma}_{A R, i}^{2} .
\end{aligned}
$$

(b) Compute $\rho^{2}$ :

$$
\begin{aligned}
& \mathrm{E}\left\{\|\mathbf{F}\|_{\mathrm{F}}^{2}\right\}=\mathrm{E}\left\{\operatorname{tr}\left(\mathbf{A B}^{T} \mathbf{B}^{*} \mathbf{A}^{H}\right\}\right), \\
& =\operatorname{tr}\left(\mathrm{E}\left\{\hat{\mathbf{G}}_{A R} \hat{\mathbf{G}}_{R B} \hat{\mathbf{G}}_{R B}^{*} \hat{\mathbf{G}}_{A R}^{H}\right\}\right) \\
& +\operatorname{tr}\left(\mathrm{E}\left\{\hat{\mathbf{G}}_{A R} \hat{\mathbf{G}}_{R B} \hat{\mathbf{G}}_{A R}^{*} \hat{\mathbf{G}}_{R B}^{H}\right\}\right) \\
& +\operatorname{tr}\left(\mathrm{E}\left\{\hat{\mathbf{G}}_{R B} \hat{\mathbf{G}}_{A R} \hat{\mathbf{G}}_{R B}^{*} \hat{\mathbf{G}}_{A R}^{H}\right\}\right) \\
& +\operatorname{tr}\left(\mathrm{E}\left\{\hat{\mathbf{G}}_{R B} \hat{\mathbf{G}}_{A R} \hat{\mathbf{G}}_{A R}^{*} \hat{\mathbf{G}}_{R B}^{H}\right\}\right) \\
& =2 M(M+1) \sum_{n=1}^{N} \sigma_{A R, n}^{2} \sigma_{R B, n}^{2} .
\end{aligned}
$$

Combining $\mathrm{E}\left\{\left\|\mathbf{F g}_{A R, i}\right\|^{2}\right\}, \mathrm{E}\left\{\left\|\mathbf{F} \mathbf{g}_{R B, i}\right\|^{2}\right\}$, and (64), $\rho^{2}$ is expressed as

$$
\rho^{2}=\frac{p_{r}}{\sum_{i=1}^{N}\left(a_{i}+b_{i}\right)+2 M(M+1) \sum_{n=1}^{N} \sigma_{A R, n}^{2} \sigma_{R B, n}^{2}},
$$

where

$$
\begin{aligned}
& a_{i}=2(M+1)\left(\tilde{\sigma}_{A R, i}^{2} p_{A, i}+\tilde{\sigma}_{R B, i}^{2} p_{B, i}\right) \\
& +M \sigma_{A R, i}^{2} \sigma_{R B, i}^{2}(M+1)(M+3)\left(\sigma_{A R, i}^{2} p_{A, i}+\sigma_{R B, i}^{2} p_{B, i}\right), \\
& b_{i}=2 M(M+1)\left(\beta_{A R, i} p_{A, i}+\beta_{R B, i} p_{B, i}\right) \sum_{n \neq i} \sigma_{A R, n}^{2} \sigma_{R B, n}^{2}
\end{aligned}
$$

We arrive at the desired result $E_{i}$ by combining (63) and (65).

\section{APPENDIX B}

PROOF OF THEOREM 6

For a given $p_{u}$, the objective function of the optimization problem $\mathcal{P}_{3}$ is an increasing function with respect to $p_{r}$, while for a given $p_{r}$, this function is an increasing function with respect to $p_{u}$; hence, the objective function is maximized when $2 N p_{u}+p_{r}=P[38]$.

Now, focusing on $\tilde{R}_{A, i}$ and substituting $2 N p_{u}+p_{r}=P$ into $\tilde{R}_{A, i}$, we have

$$
\tilde{R}_{A, i}=\frac{1}{2} \log _{2}\left(1+\frac{1}{a+\frac{b}{p_{u}}+\frac{c}{d-p_{u}}}\right),
$$

where $a=\frac{1}{M}\left(\frac{\beta_{R B, i}+4 \beta_{A R, i}}{\sigma_{R B, i}^{2}}+\frac{\beta_{A R, i}}{\sigma_{A R, i}^{2}}\right)+$ $\frac{1}{M} \sum_{j \neq i}\left(\frac{\beta_{A R, j}}{\sigma_{R B, i}^{2}}+\frac{\sigma_{A R, j}^{4} \sigma_{R B, j}^{2} \beta_{A R, i}}{\sigma_{A R, i}^{4} \sigma_{R B, i}^{4}}\right)+$ $\frac{1}{M} \sum_{j \neq i}\left(\frac{\beta_{R B, j}}{\sigma_{R B, i}^{2}}+\frac{\sigma_{A R, j}^{2} \sigma_{R B, j}^{4} \beta_{A R, i}}{\sigma_{A R, i}^{4} \sigma_{R B, i}^{4}}\right), \quad b=\frac{1}{M \sigma_{R B, i}^{2}}$, $c=\frac{1}{2 M N \sigma_{A R, i}^{4} \sigma_{R B, i}^{4}} \sum_{n=1}^{N}\left(\sigma_{A R, n}^{2} \sigma_{R B, n}^{2}\left(\sigma_{A R, n}^{2}+\sigma_{R B, i}^{2}\right)\right)$, and $d=\frac{P}{2 N}$. 
Taking the second derivative with respect to $p_{u}$ yields

$$
\begin{aligned}
\frac{\partial^{2} \tilde{R}_{A, i}}{\partial p_{u}^{2}} & =-\frac{b^{2}\left(2 c d^{2}+(1+2 a)\left(d-p_{u}\right)^{3}\right)\left(d-p_{u}\right)}{2 \ln 2 f^{2} g^{2}} \\
& -\frac{c\left(c+2 a c+2 a(1+a)\left(d-p_{u}\right)\right) p_{u}^{4}}{2 \ln 2 f^{2} g^{2}} \\
& -\frac{b p_{u}\left(c^{2} d^{2}+a(1+a)\left(d-p_{u}\right)^{4}\right)}{\ln 2 f^{2} g^{2}} \\
& -\frac{b p_{u}(1+2 a) c\left(d-p_{u}\right)\left(d^{2}-d p_{u}+p_{u}^{2}\right)}{\ln 2 f^{2} g^{2}}<0,
\end{aligned}
$$

where $f=b\left(d-p_{u}\right)+\left(c+a\left(d-p_{u}\right)\right) p_{u}$ and $g=f+$ $\left(d-p_{u}\right) p_{u}$ Thus, $\tilde{R}_{A, i}$ is a strictly concave function with respect to $p_{u}$. Since nonnegative weighted sums preserve convexity [39], the objective function $\frac{\tau_{c}-\tau_{p}}{\tau_{c}} \sum_{i=1}^{N}\left(\tilde{R}_{A, i}+\tilde{R}_{B, i}\right)$ is also a strictly concave function with respect to $p_{u}$. Recall that the constraints of the optimization problem $\mathcal{P}_{3}$ are all affine functions, and hence $\mathcal{P}_{3}$ is a convex optimization problem.

\section{REFERENCES}

[1] H. Q. Ngo, H. A. Suraweera, M. Matthaiou, and E. G. Larsson, "Multipair full-duplex relaying with massive arrays and linear processing," IEEE J. Sel. Areas Commun., vol. 32, no. 9, pp. 1721-1737, Oct. 2014.

[2] H. A. Suraweera, H. Q. Ngo, T. Q. Duong, C. Yuen, and E. G. Larsson, "Multi-pair amplify-and-forward relaying with very large antenna arrays," in Proc. IEEE ICC, June 2013, pp. 4635-4640.

[3] G. Kramer, M. Gastpar, and P. Gupta, "Cooperative strategies and capacity theorems for relay networks," IEEE Trans. Inf. Theory, vol. 51, no. 9, pp. 3037-3063, Sept. 2005.

[4] Q. Wang and Y. Jing, "Performance analysis and scaling law of MRC/MRT relaying with CSI error in multi-pair massive MIMO systems", IEEE Trans. Wireless Commun., vol. 16, no. 9, pp. 5882-5896, Sept. 2017.

[5] F. Gao, T. Cui, and A. Nallanathan, "On channel estimation and optimal training design for amplify and forward relay network", IEEE Trans. Wireless Commun., vol. 7, no. 5, pp. 1907-1916, May 2008.

[6] R. Zhang, Y-C. Liang, C. C. Chai, and S. Cui, "Optimal beamforming for two-way multi-antenna relay channel with analogue network coding," IEEE J. Sel. Areas Commun., vol. 27, no. 5, pp. 699-712, Jun. 2009.

[7] K.-J. Lee, H. Sung, E. Park, and I. Lee, "Joint optimization for one and two-way MIMO AF multiple-relay systems," IEEE Trans. Wireless Commun., vol. 9, no. 12, pp. 3671-3681, Dec. 2010.

[8] G. Amarasuriya, C. Tellambura, and M. Ardakani, "Two-way amplifyand-forward multiple-input multiple-output relay networks with antenna selection," IEEE J. Sel. Areas Commun., vol. 30, no. 8, pp. 1513-1529, Sept. 2012.

[9] R. Vaze and R.W. Heath Jr., "On the capacity and diversity-multiplexing tradeoff of the two-way relay channel," IEEE Trans. Inf. Theory, vol. 57, no. 7, pp. 4219-4234, July 2011.

[10] T. Cui, F. Gao, T. Ho, and A. Nallanathan, "Distributed space-time coding for two-way wireless relay networks", IEEE Trans. Signal Process., vol. 57, no. 2, pp. 658-671, Feb. 2009.

[11] F. Gao, R. Zhang, and Y.-C. Liang, "Optimal channel estimation and training design for two-way relay networks", IEEE Trans. Commun., vol. 57 , no. 10, pp. 3024-3033, Oct. 2009.

[12] F. Gao, R. Zhang, and Y.-C. Liang, "Channel estimation for OFDM modulated two-way relay networks," IEEE Trans. Signal Process., vol. 57, no. 11, pp. 4443-4455, Nov. 2009.

[13] H. Cui, L. Song, and B. Jiao, "Multi-pair two-way amplify-and-forward relaying with very large number of relay antennas," IEEE Trans. Wireless Commun., vol. 13, no. 5, pp. 2636-2645, May 2014.

[14] S. Jin, X. Liang, K.-K. Wong, X. Gao, and Q. Zhu, "Ergodic rate analysis for multipair massive MIMO two-way relay networks," IEEE Trans. Wireless Commun., vol. 14, no. 3, pp. 1480-1491, Mar. 2015.

[15] C. Kong, C. Zhong, M. Matthaiou, E. Björnson, and Z. Zhang, "Multipair two-way AF relaying systems with massive arrays and imperfect CSI," in Proc. IEEE ICASSP, Mar. 2016, pp. 3651-3655.
[16] C. Kong, A. Mezghani, C. Zhong, A. Swindlehurst, and Z. Zhang, "Multipair massive MIMO relaying systems with one-bit ADCs and DACs," IEEE Trans. Signal Process., vol. 66, no. 11, pp. 2984-2997, Nov. 2018.

[17] C. Kong, A. Mezghani, C. Zhong, A. Swindlehurst, and Z. Zhang, "Nonlinear precoding for multipair relay networks with one-bit ADCs and DACs," IEEE Signal Process. Lett., vol. 25, no. 2, pp. 303-307, Feb. 2018.

[18] S. Sima and W. Chen, "Joint network and dirty-paper coding for multiway relay networks with pairwise information exchange," in Proc. IEEE GLOBECOM, Dec. 2014, pp. 1565-1570.

[19] R. S. Ganesan, H. Al-Shatri, A. Kuehne, T. Weber, and A. Klein, "Pairaware interference alignment in multi-user two-way relay networks," IEEE Trans. Wireless Commun., vol. 12, no. 8, pp. 3662-3671, Aug. 2013.

[20] T. L. Marzetta, "Noncooperative cellular wireless with unlimited numbers of base station antennas," IEEE Trans. Wireless Commun., vol. 9, no. 11, pp. 3590-3600, Nov. 2010.

[21] M. Yemini, A. Zappone, E. Jorswieck, and A. Leshem, "Energy efficient bidirectional massive MIMO relay beamforming," IEEE Signal Process. Lett., vol. 24, no. 7, pp. 1010-1014, July 2017.

[22] Z. Zhang, Z. Chen, M. Shen, and B. Xia, "Spectral and energy efficiency of multipair two-way full-duplex relay systems with massive MIMO," IEEE J. Sel. Areas Commun., vol. 34, no. 4, pp. 848-863, Apr. 2016.

[23] J. Feng, S. Ma, G. Yang, and B. Xia, "Power scaling of full-duplex two-way massive MIMO relay systems with correlated antennas and MRC/MRT processing," IEEE Trans. Wireless Commun., vol. 16, no. 7, pp. 4738-4753, July 2017.

[24] H. Q. Ngo and E. G. Larsson, "Spectral efficiency of the multipair twoway relay channel with massive arrays," in Proc. IEEE ACSSC, Nov. 2013, pp. 275-279.

[25] Y. Dai and X. Dong, "Power allocation for multi-pair massive MIMO two-way AF relaying with linear processing," IEEE Trans. Wireless Commun., vol. 15, no. 9, pp. 5932-5946, Sept. 2016.

[26] C. Kong, C. Zhong, M. Matthaiou, E. Björnson, and Z. Zhang, "Multipair two-way half-duplex DF relaying with massive arrays and imperfect CSI," IEEE Trans. Wireless Commun., vol. 17, no. 5, pp. 3269-3283, Mar. 2018.

[27] H. Q. Ngo, E. G. Larsson, and T. L. Marzetta, "Energy and spectral efficiency of very large multiuser MIMO systems," IEEE Trans. Commun., vol. 61, no. 4, pp. 1436-1449, Apr. 2013.

[28] C. Wang, T. C.-K. Liu, and X. Dong, "Impact of channel estimation error on the performance of amplify-and-forward two-way relaying," IEEE Trans. Veh. Technol., vol. 61, no. 3, pp. 1197-1207, Mar. 2012.

[29] J. Hoydis, S. ten Brink, and M. Debbah, "Massive MIMO in the UL/DL of cellular networks: How many antennas do we need?", IEEE J. Sel. Areas Commun., vol. 31, no. 2, pp. 160-171, Feb. 2013.

[30] M. Avriel and A. C. Williams, "Complementary geometric programming," SIAM J. Appl. Math., vol. 19, no. 1, pp. 125-141, July 1970.

[31] M. Grant and S. Boyd, CVX: Matlab software for disciplined convex programming, June 2015. [Online]. Available: http://cvxr.com/cvx/

[32] A. Mutapcic, K. Koh, S. Kim, and S. Boyd, A Matlab Toolbox for Geometric Programming, May 2006. [Online]. Available: http://www.stanford.edu/ boyd/ggplab/ggplab.pdf

[33] P. C. Weeraddana, M. Codreanu, M. Latva-aho, and A. Ephremides, "Resource allocation for cross-layer utility maximization in wireless networks," IEEE Trans. Veh. Technol., vol. 60, no. 6, pp. 2790-2809, July 2011

[34] Y. Nesterov and A. Nemirovsky, Interior Point Polynomial Methods in Convex Programming. SIAM Press, 1994.

[35] B. R. Marks and G. P. Wright, "A general inner approximation algorithm for nonconvex mathematical programs," Oper. Res., vol. 26, no. 4, pp. 681-683, Jul./Aug. 1978.

[36] S. Boyd, S. J. Kim, L. Vandenberghe, and A. Hassibi, "A tutorial on geometric programming," Optim. Eng., vol. 8, no. 1, pp. 67-127, Apr. 2007.

[37] S. Boyd, Sequential Convex Programming, 2007. [Online]. Available: http://www.stanford.edu/class/ee364b/lectures/seq_slides.pdf

[38] H. Q. Ngo, M. Matthaiou, and E. G. Larsson, "Massive MIMO with optimal power and training duration allocation," IEEE Wireless Commun. Lett., vol, 3, no. 6, pp. 605-608, Dec. 2014.

[39] S. Boyd and L. Vandenberghe, Convex Optimization. Cambridge, UK: Cambridge University Press, 2004. 


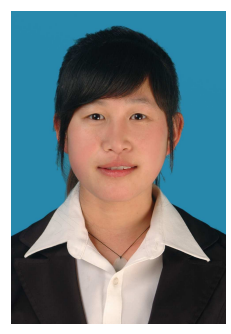

Chuili Kong (S'15) received the B.S. degree in electronics and information engineering from the Dalian University of Technology, Dalian, China, in 2013. She is currently working towards the Ph.D. degree in information and communication engineering at the Zhejiang University. From October 2016 to October 2017, she was a visiting scholar at University of California, Irvine. She was an exemplary reviewer for IEEE TRANSACTIONS ON WIRELESS COMMUNICATIONS in 2017. Her research interests include massive MIMO systems and relaying communica-

tions.

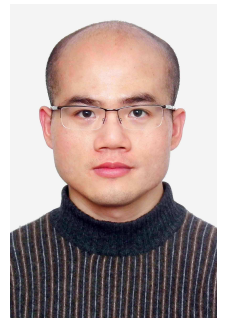

Caijun Zhong (S'07-M'10-SM'14) received the B.S. degree in Information Engineering from the Xi'an Jiaotong University, Xi'an, China, in 2004, and the M.S. degree in Information Security in 2006, $\mathrm{Ph} . \mathrm{D}$. degree in Telecommunications in 2010, both from University College London, London, United Kingdom. From September 2009 to September 2011, he was a research fellow at the Institute for Electronics, Communications and Information Technologies (ECIT), Queens University Belfast, Belfast, UK. Since September 2011, he has been with Zhejiang University, Hangzhou, China, where he is currently a Professor. His research interests include massive MIMO systems, wireless power transfer, backscatter communications and NOMA

Dr. Zhong is an Editor of the IEEE TRANS ACTIONS ON WIRELESS COMMUNICATIONS, SCIENCE CHINA: INFORMATION SCIENCE, and several other international journals. He was an Editor of the IEEE COMMUNICATIONS LETTERS from 2014-2018. He is the recipient of the 2013 IEEE ComSoc Asia-Pacific Outstanding Young Researcher Award. He has received Best Paper Awards at the WCSP 2013 and IEEE ICCC 2018.

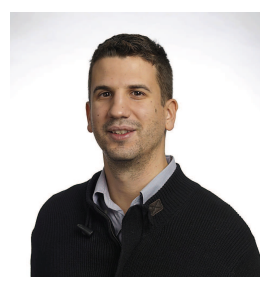

Michail Matthaiou (S'05-M'08-SM'13) was born in Thessaloniki, Greece in 1981. He obtained the Diploma degree (5 years) in Electrical and Computer Engineering from the Aristotle University of Thessaloniki, Greece in 2004. He then received the M.Sc. (with distinction) in Communication Systems and Signal Processing from the University of Bristol, U.K. and Ph.D. degrees from the University of Edinburgh, U.K. in 2005 and 2008, respectively. From September 2008 through May 2010, he was with the Institute for Circuit Theory and Signal Processing, Munich University of Technology (TUM), Germany working as a Postdoctoral Research Associate. He is currently a Reader (equivalent to Associate Professor) in Multiple-Antenna Systems at Queen's University Belfast, U.K. after holding an Assistant Professor position at Chalmers University of Technology, Sweden. His research interests span signal processing for wireless communications, massive MIMO, hardware-constrained communications, and performance analysis of fading channels.

Dr. Matthaiou and his coauthors received the 2017 IEEE Communications Society Leonard G. Abraham Prize. He is currently holding the prestigious 2018/2019 Royal Academy of Engineering/The Leverhulme Trust Senior Research Fellowship and will receive the 2019 EURASIP Early Career Award. His team was the Grand Winner of the 2019 Mobile World Congress Challenge. He was the recipient of the 2011 IEEE ComSoc Best Young Researcher Award for the Europe, Middle East and Africa Region and a corecipient of the 2006 IEEE Communications Chapter Project Prize for the best M.Sc. dissertation in the area of communications. He has co-authored papers that received best paper awards at the 2018 IEEE WCSP and 2014 IEEE ICC and was an Exemplary Reviewer for IEEE COMMUNICATIONS LETTERS for 2010. In 2014, he received the Research Fund for International Young Scientists from the National Natural Science Foundation of China. In the past, he was an Associate Editor for the IEEE TRANSACTIONS ON COMMUNICATIONS, Associate Editor/Senior Editor for IEEE COMMUNICATIONS LETTERS and was the Lead Guest Editor of the special issue on "Large-scale multiple antenna wireless systems" of the IEEE JOURNAL On SELECTED AREAS IN COMMUNICATIONS.

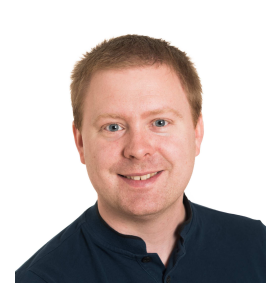

E mil Björnson (S'07-M'12-SM'17) received the M.S. degree in engineering mathematics from Lund University, Sweden, in 2007, and the Ph.D. degree in telecommunications from the KTH Royal Institute of Technology, Sweden, in 2011. From 2012 to 2014, he held a joint post-doctoral position at the Alcatel-Lucent Chair on Flexible Radio, SUPELEC, France, and the KTH Royal Institute of Technology. He joined Linköping University, Sweden, in 2014, where he is currently an Associate Professor and a tems. Docent with the Division of Communication Sys-

He has authored the textbooks Optimal Resource Allocation in Coordinated Multi-Cell Systems (2013) and Massive MIMO Networks: Spectral, Energy, and Hardware Efficiency (2017). He is dedicated to reproducible research and has made a large amount of simulation code publicly available. He performs research on MIMO communications, radio resource allocation, machine learning for communications, and energy efficiency. Since 2017, he has been on the Editorial Board of the IEEE TRANSACTIONS ON COMMUNICATIONS and the IEEE TRANSACTIONS ON GREEN COMMUNICATIONS AND NETWORKING since 2016.

$\mathrm{He}$ has performed MIMO research for over ten years and has filed more than ten MIMO related patent applications. He has received the 2014 Outstanding Young Researcher Award from IEEE ComSoc EMEA, the 2015 Ingvar Carlsson Award, the 2016 Best Ph.D. Award from EURASIP, the 2018 IEEE Marconi Prize Paper Award in Wireless Communications, the 2019 EURASIP Early Career Award, and the 2019 IEEE Communications Society Fred W. Ellersick Prize. He also co-authored papers that received Best Paper Awards at the conferences, including WCSP 2009, the IEEE CAMSAP 2011, the IEEE WCNC 2014, the IEEE ICC 2015, WCSP 2017, and the IEEE SAM 2014.

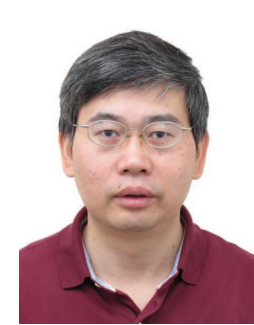

Zhaoyang Zhang (M'00) received his Ph.D. degree in communication and information systems from Zhejiang University, Hangzhou, China, in 1998. Since then he has been with the College of Information Science and Electronic Engineering, Zhejiang University, where he became a full professor in 2005. He has a wide variety of research interests including information theory and coding theory, signal processing for communications and in networks, computation-and-communication theoretic analysis, etc. He was a co-recipient of four international conference Best Paper / Best Student Paper Awards. He is currently serving as Editor for IEEE Transactions on Wireless Communications, IEEE Transactions on Communications and some other international journals. He served as General Chair, TPC Co-Chair or Symposium Co-Chair for many international conferences and workshops like ChinaCOM 2008, ICUFN 2011/2012/2013, WCSP 2013, Globecom 2014 Wireless Communications Symposium, and VTC-Fall 2017 Workshop HMWC, etc. 